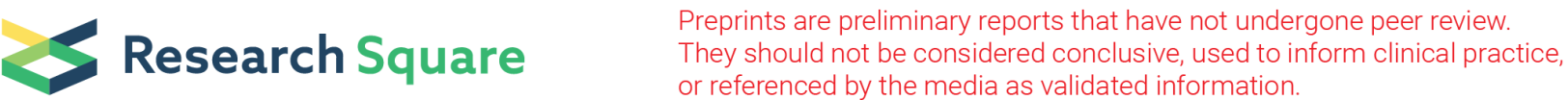

\section{WDR31 is a novel ciliopathy protein displaying functional redundancy with GTPase-activating proteins ELMOD and RP2 in recruiting BBSome to cilium}

\section{Sebiha Cevik}

Abdullah Gul University

\section{Lama Alabdi}

King Faisal Specialist Hospital and Research Center, https://orcid.org/0000-0001-8177-4048

\section{Xiaoyu Peng}

Tongji University

\section{Tina Beyer}

University of Tuebingen; Institute for Ophthalmic Research

\section{Atiyye Zorluer}

Abdullah Gul University

\section{Mustafa Pir}

Abdullah Gul University https://orcid.org/0000-0002-4645-7626

\section{Ferhan Yenisert}

Abdullah Gul University

\section{Ranad Shaheen}

King Faisal Specialist Hospital and Research Center, https://orcid.org/0000-0002-2590-1759

\section{Franziska Woerz}

University of Tuebingen

\section{Felix Hoffmann}

University of Tuebingen

\section{Betul Altunkaynak}

Abdullah Gul University

\section{Betul Pir}

Abdullah Gul University

\section{Karsten Boldt}

Institute for Ophthalmic Research Centre for Ophthalmology, University of Tuebingen https://orcid.org/0000-0002-2693-689X

\section{Asli Karaman}

Istanbul Medeniyet University

\section{Miray Cakiroglu}


Istanbul Medeniyet University https://orcid.org/0000-0002-4289-8344

\section{Sadik Oner}

Istanbul Medeniyet University

\section{Ying Cao}

Department of Clinical Laboratory Medicine of Shanghai Tenth People's Hospital, Tongji University

School of Life Sciences and Technology https://orcid.org/0000-0001-7290-0655

\section{Marius Ueffing}

University of Tuebingen

\section{Fowzan Alkuraya}

King Faisal Specialist Hospital \& Research Centre https://orcid.org/0000-0003-4158-341X

Oktay Kaplan ( $\square$ oktaykaplan@gmail.com )

Abdullah Gul University https://orcid.org/0000-0002-8733-0920

\section{Article}

Keywords: Defective Cilia, Homozygous Truncating Variant, Evolutionarily Conserved Protein, Cilia Morphology, IFT Trafficking, BBSome Trafficking

Posted Date: July 12th, 2021

DOI: https://doi.org/10.21203/rs.3.rs-622797/v1

License: (c) (1) This work is licensed under a Creative Commons Attribution 4.0 International License. Read Full License 

activating proteins ELMOD and RP2 in recruiting BBSome to cilium

3 Sebiha Cevik $^{1 *}$, Lama Alabdi ${ }^{2,5^{*}}$, Xiaoyu Peng ${ }^{3}$, Tina Beyer $^{4}$, Atiyye Zorluer $^{1}$, Mustafa S. Pir ${ }^{1}$,

4 Ferhan Yenisert ${ }^{1}$, Ranad Shaheen ${ }^{2}$, Franziska Woerz ${ }^{4}$, Felix Hoffmann ${ }^{4}$, Betul Altunkaynak ${ }^{1}$,

5 Betul Pir ${ }^{1}$, Karsten Boldt ${ }^{4}$, Asli Karaman ${ }^{7}$, Miray Cakiroglu ${ }^{7}$, S. Sadik Oner ${ }^{6,7}$, Ying Cao ${ }^{3}$,

6 Marius Ueffing 4 , Fowzan S. Alkuraya ${ }^{2}$, Oktay I. Kaplan ${ }^{1} \uparrow$

7 1- Rare Disease Laboratory, School of Life and Natural Sciences, Abdullah Gul University,

8 Kayseri, Turkey

9 2- Department of Translational Genomics, Center for Genomic Medicine, King Faisal Specialist

10 Hospital and Research Center, Riyadh, Saudi Arabia.

11 3- School of Life Sciences and Technology, Tongji University, Shanghai 200092, China

12 4- Institute for Ophthalmic Research, Centre for Ophthalmology, University of Tuebingen,

13 Elfriede-Aulhorn-Strasse 7, D-72076 Tuebingen, Germany.

14 5- Department of Zoology, College of Science, King Saud University, Riyadh, Saudi Arabia

15 6- Goztepe Prof. Dr. Suleyman Yalcin City Hospital, Istanbul, Turkey

16 7- Science and Advanced Technology Application and Research Center, Istanbul Medeniyet

17 University, Istanbul

$18 *$ These two authors contributed equally to this work

$19 †$ Correspondence to oktay.kaplan@agu.edu.tr 


\section{Abstract}

26 The term "ciliopathy" refers to a group of over 35 rare disorders characterized by defective cilia

27 and many overlapping clinical features, such as hydrocephalus, cerebellar vermis hypoplasia,

28 polydactyly, and retinopathy. Even though many genes have been implicated in ciliopathies, the

29 genetic pathogenesis in certain cases remains still undisclosed. Here, we identified a homozygous

30 truncating variant in WDR31 in a patient with a typical ciliopathy phenotype encompassing

31 congenital hydrocephalus, polydactyly, and renal agenesis. WDR31 is an evolutionarily conserved

32 protein that localizes to the cilium and cilia-related compartment. Analysis from zebrafish supports

33 the role of WDR31 in regulating the cilia morphology. The CRISPR/Cas9 knock-in (p.Arg261del)

34 C. elegans model of the patient variant (p.Arg268*) reproduced several cilia-related defects

35 observed in wdr-31 null mutants. Mechanistic analysis from C. elegans revealed that WDR-31

36 functions redundantly with ELDM-1 (ELMOD protein) and RPI-2 (RP2) to regulate the IFT

37 trafficking through controlling the cilia entry of the BBSome. This work revealed WDR31 as a

38 new ciliopathy protein that regulates IFT and BBSome trafficking. 


\section{Introduction}

48 Cilia are structurally and functionally distinct cellular projections, consisting of a microtubule-

49 based axoneme extending from a centriole-derived basal body anchored at the plasma membrane.

50 Cilia consist of multiple sub-compartments (basal body, transition zone, and ciliary tip) that

51 display different protein compositions and structure (Blacque and Sanders, 2014; Rosenbaum and

52 Witman, 2002; Satir and Christensen, 2007). Motile cilia mediate the movement of unicellular

53 organisms such as Chlamydomonas reinhardtii or are involved in fluid movement across a tissue

54 surface (Silflow and Lefebvre, 2001; Sleigh, 1989). Non-motile cilia, also known as primary cilia,

55 possess mechanosensory, chemosensory, and osmosensory functions, and coordinate a range of

56 extrinsic signaling pathways involved in cellular behavior, tissue development, and homeostasis

57 such as those mediated by Hedgehog (Hh), Wnt, and receptor tyrosine kinase ligands (e.g. PDGF $\alpha$ )

58 (Anvarian et al., 2019; Bloodgood, 2009; Nachury, 2014; Scholey, 2007).

59 The relation between cilia and human disorders has led to a greater understanding of their

60 importance for human health. Both motile and primary cilia have been linked to the heterogeneous

61 class of diseases known as ciliopathies, including Joubert Syndrome, Meckel Syndrome (MKS),

62 and Nephronophthisis (NPHP). Owing to the presence of cilia on most cell types, ciliary defects

63 result in varying multiorgan phenotypes such as kidney defects, retinitis pigmentosa, pancreatic

64 cysts, hearing loss, congenital heart disease, and polydactyly (Reiter and Leroux, 2017; Wheway

65 et al., 2019).

66 Over the last twenty years, there has been a large effort to reveal the molecular composition of

67 cilia and its sub-compartments using several independent approaches, including clinical genomics,

68 proteomics, functional genomics, and bioinformatics (Arnaiz et al., 2009; Avidor-Reiss et al., 
69 2004; Blacque et al., 2005; Breslow et al., 2018; Choksi et al., 2014; Jensen et al., 2016, p.

70 26595381; Lambacher et al., 2016; Li et al., 2004; Mick et al., 2015; Piasecki et al., 2010; Ruiz

71 García et al., 2019; Shaheen et al., 2016; Shamseldin et al., 2020; Sigg et al., 2017; UK10K Rare

72 Diseases Group et al., 2016; van Dam et al., 2019). Many proteins that make up the cilium, as well

73 as many proteins that regulate cilia biology, have been identified. These collective efforts have

74 resulted in the identification of 302 genes that are certain to be involved in cilia biogenesis, as well

75 as over 180 ciliopathy genes. CiliaCarta estimates the total number of the ciliary genes to be about

761200 genes, implying that many more ciliary proteins and ciliopathy genes are yet to be discovered.

77 Indeed, the genetic diagnosis of many ciliopathy disorders is still unknown (Shamseldin et al.,

78 2020, 2020; SYSCILIA Study Group et al., 2013; van Dam et al., 2019; Wheway et al., 2019).

79 Owing to the lack of protein-synthesizing machinery in cilia, structural components of cilia and

80 cilia cargos must be transported to cilia in order to construct and sustain cilia, and mutations in

81 genes involved in ciliary trafficking are commonly seen in ciliopathies. Cilia has a one-of-a-kind

82 protein delivery system called intraflagellar transport (IFT). IFT is made up of multisubunit protein

83 complexes that travel bidirectionally along the cilia. The IFT complex contains two sub-

84 complexes, IFT-A and IFT-B, consisting of 6 and 16 protein subunits, respectively (Prevo et al.,

85 2017). The IFT-B sub-complex and Kinesin-2 motors mediate the motility of IFT and IFT cargos

86 from the base of the cilia to the tip of the cilia (anterograde IFT), while the IFT-A sub-complex

87 and the cytoplasmic dynein-2 motor facilitate the retrograde IFT transport (from the cilia tip to the

88 base of the cilia) (Blacque, 2008; Rosenbaum and Witman, 2002). The IFT-A is involved in the

89 transport of certain membrane proteins into cilia (Lee et al., 2008; Liem et al., 2012;

90 Mukhopadhyay et al., 2010). Mutations in genes encoding IFT components lead to defects in cilia 
91 formation in all examined organisms, indicating the importance of IFT for cilia assembly (Pazour 92 et al., 2000; Prevo et al., 2017).

93 Bardet-Biedl syndrome (BBS) was classified as a ciliopathy in 2003, and eight of the highly 94 conserved Bardet-Biedl syndrome proteins (BBS1, BBS2, BBS4, BBS5, BBS7, BBS8, BBS9, and 95 BBIP10) establish a stable protein complex called the BBSome that undergoes IFT. Work from a 96 range of organisms implicates the BBSome in a variety of cilia related process including acting as 97 a cargo adaptor for removing proteins from cilia and as a regulator of the assembly and stability 98 of IFT trains (Ansley et al., 2003; Lechtreck et al., 2009; Loktev et al., 2008; Nachury et al., 2007;

99 Nozaki et al., 2019; Ou et al., 2007, 2005; Wei et al., 2012; Williams et al., 2014; Xu et al., 2015; 100 Ye et al., 2018). For example, in the nematode C. elegans mutants lacking bbs-7 or bbs-8, 101 detachment of IFT-A and IFT-B in the amphid cilia was reported in the anterograde direction (Ou 102 et al., 2005). Analysis with a hypomorphic mutant (bbs-1) revealed that BBSome is involved in 103 attaching IFT-B components to the retrograde IFT machinery at the ciliary tips in the channel cilia 104 of C. elegans (Wei et al., 2012).

105 To identify new ciliopathy genes, we focused on our single-cell RNA-seq data that compared 106 expression profiles of ciliated cells with those of non-ciliated cells in the nematode C. elegans, and 107 this work revealed novel cilia genes, including WDR31 (manuscript in preparation). Our gene 108 discovery approach in combination with the use of clinical genetic testing and experimental 109 validations identified WDR31 (WD repeat domain 31) as a novel ciliopathy gene encoding a highly 110 conserved cilia-associated protein. Our work from Zebrafish and C. elegans provides significant 111 insight into the function of WDR31 in cilia biogenesis. First, our work from C. elegans revealed 112 that WDR-31 functions redundantly with two GTPase activating proteins (GAPs) ELMD-1 (the 113 sole ortholog of the human ELMOD proteins) and RPI-2 (human retinitis pigmentosa 2 
114 orthologue) to control the cilia morphology. Second, knocking out $w d r-31$ along with elmd-1 or 115 elmd-1;rpi-2 causes IFT trafficking to be disrupted, resulting in ciliary tip accumulations of IFT116 B components and the OSM-3/KIF17 motor, as well as significantly reduced BBSome recruitment 117 to cilia. Taken together, WDR31 is a new ciliopathy-associated protein that exhibits functional 118 redundancy with two GAP proteins (ELMOD and RP2) in regulating cilia morphology and IFT 119 trafficking.

121 Material and Methods

\section{C. elegans strains, maintenance and genetic crossing}

123 For strain maintenance and genetic crosses, a standard procedure was followed, as described by 124 Sidney Brenner in 1974 (Brenner, 1974). After genetic cross with a marker to generate single, 125 double and triple mutants, we used the PCR strategy to trace the mutations in following mutants: $126 w d r-31(T 05 A 8.5)(t m 10423) I I . ; \quad w d r-31(t u r 003) I I$.; $w d r-31(s y b 1568) I I . ; \quad n p h p-4(t m 925) V . ; \quad m k s-$ 127 6(gk674)I.; elmd-1(syb630) III.; RB1550 rpi-2(K08D12.2)(ok1863) IV.; and bbs-8(nx77) V. 128 Primers can be found in Table S1.

\section{Lipophilic fluorescent dye-uptake assay and rescue analysis}

131 Healthy mixed-staged animals were collected using M9 buffer (3 g/L KH2PO4, 6 g/L Na2HPO4, $1325 \mathrm{~g} / \mathrm{L} \mathrm{NaCl}, 1 \mathrm{mM} \mathrm{MgSO} 4)$, centrifuged for 1 minute at $2000 \mathrm{rpm}$, and washed twice with M9 133 buffer to remove any bacterial contaminations. Worms were incubated for 45-60 minutes at room 134 temperature in an M9 buffer containing lipophilic dye (1:200 dilution in M9, Invitrogen ${ }^{\mathrm{TM}}$ 135 Vybrant ${ }^{\mathrm{TM}}$ DiI Cell-Labeling Solution) (Herman and Hedgecock, 1990). The worms were then 136 washed twice with M9 before being moved to a new NGM plate. Wild type was always included 
137 in Dye filling assay, and the dye uptake control for the wild type was performed under a stereotype 138 fluorescence microscope, followed by imaging with the fluorescence upright microscope. For the 139 rescue experiment, N2;turEx24[arl-13p::GFP::elmd-1 (C56G7.3)::unc-54 3'UTR +rol-6\} 140 (OIK1045) were crossed into wdr-31(T05A8.5)(tm10423)II., elmd-1(syb630) II, double and wdr141 31(T05A8.5)(tm10423)II., elmd-1(syb630) II, rpi-2(K08D12.2)(ok1863) IV. triple mutants. 142 N2;turEx21 [arl-13p::wdr-31 (T05A8.5)::GFP::unc-54 3'UTR +rol-6\} (1 ng) (OIK1042) were 143 mated with T05A8.5(syb1568)II., elmd-1(syb630) II, rpi-2(K08D12.2)(ok1863) IV. triple mutants. 144 Plasmid (arl-13p::wdr-31 (T05A8.5)::GFP::unc-54 3'UTR) was directly microinjected into triple 145 mutant T05A8.5(syb1568)II., elmd-1(syb630) II, rpi-2(K08D12.2)(ok1863) IV (1 ng). dpy146 5(e907);nxEx386[rpi-2::gfp $+d p y-5(+)]$ (MX352) were crossed into $w d r-$ 147 31(T05A8.5)(tm10423)II., elmd-1(syb630) II, rpi-2(K08D12.2)(ok1863) IV. triple mutants. Three 148 independent Dye uptake assays were performed, and fluorescence filters were set for GFP and 149 Texas Red, followed by fluorescence imaging. Dye uptake of mutants with corresponding 150 transgenics strains was compared to that of non-transgenic strains in the same rescue plates (Fig. 151 S2C, D).

\section{Generation of mutants using CRISPR/Cas9 in the nematode Caenorhabditis elegans}

154 To generate $w d r-31(T 05 A 8.5)$ (tur003) allele, three sgRNAs targeting C. elegans T05A8.5 (human 155 WDR-31) were chosen using an online tool, Benchling [Biology Software] (2019), followed by 156 ordering complementary oligonucleotides (Macrogen, South Korea), and cloning of sgRNAs into 157 an empty sgRNA vector pRB1017. The successful sgRNA insert was confirmed with colony PCR, 158 followed by plasmid isolation. Three sgRNAs (each $50 \mathrm{ng} / \mu \mathrm{l}$ ) were injected into the gonads of 159 wild type together with pDD162 (Peft-3::Cas9; $15 \mathrm{ng} / \mu \mathrm{l}$ plasmid pRF4) and pRF4 (50 ng/ $\mu \mathrm{l}$ 
plasmid pRF4) (Dickinson et al., 2013). F1s with the roller phenotype were identified, and after they generated enough progenies, the PCR technique was used to identify the F1 generation

162 bearing the predicted deletion, as well as the homozygosity of the allele mutation. The PCR 163 products from knockout animals were then sent to the Sanger sequencing (Macrogen, South

164 Korea). $w d r-31$ (tur003)II. mutants have 1276-bp deletion covering a huge part of exon II (297 bp 165 out of $359 \mathrm{bp}$ ) and whole exon III, IV, and exon V. This is likely a null allele of $w d r-31$. sgRNA sequences can be found in Table S1.

\section{Generation of transgenic strains and strain list for $C$. elegans}

To generate transgenic lines for localization, rescue experiments, and expression patterns, we generated the following transgenic animals via microinjections.

T05A8.5(syb1568)II., elmd-1(syb630) II, rpi-2(K08D12.2)(ok1863) IV. (1 ng)

OIK1044 N2;turEx23[elmd-1p::GFP::elmd-1 (C56G7.3)::unc-54 3'UTR +rol-6\} (5 ng)

OIK1045 N2;turEx24[arl-13p::GFP::elmd-1 (C56G7.3)::unc-54 3’UTR +rol-6\} (5 ng)

OIK1046 N2;turEx25[elmd-1p(C56G7.3)::GFP::unc-54 3'UTR +rol-6\} (50 ng)

OIK1047 N2;turEx26[wdr-31 (T05A8.5)p::GFP::unc-54 3’UTR +rol-6\} (50 ng)

177 The rol-6 plasmid (50 ng/ $\mu 1$ plasmid pRF4) was co-injected as the co-transformation marker. In 178 brief, the plasmids were delivered by microinjections into the gonads of 1-day adult worms. 179 Worms were initially transferred onto a 2.5\% agarose pad prior (Halocarbon oil, Sigma: 9002-83- 
183 Wild Type and mutant alleles: N2; FX30333; wdr-31(T05A8.5)(tm10423)II.; OIK393 184 T05A8.5(tur003)II.; $\quad$ PHX1568 T05A8.5(syb1568)II.; $\quad$ nphp-4(tm925)V.; $\quad$ mks-6(gk674)I.; 185 PHX630, elmd-1(syb630) III.; RB1550 rpi-2(K08D12.2)(ok1863) IV.; MX52, bbs-8(nx77) V. We 186 obtained $w d r-31(T 05 A 8.5)(t m 10423) I I$. (FX30333) mutant allele, which has a 160-bp deletion, 187 causing a frameshift, from the National Bioresource Project, Japan. The wdr188 31(T05A8.5)(tm 10423)II. were outcrossed to wild type four times. The Caenorhabditis Genetics 189 Center (CGC), Minnesota, USA, provided the RB1550 rpi-2(K08D12.2)(ok1863) mutant, and the 190 rpi-2(ok1863) allele contains 1143-bp deletion that removes a large segment of exon III, exon IV, 191 and some portion of exon V. T05A8.5(syb1568)II. has 1888-bp deletion, deleting all exons except 192 exon I (Fig. S3B). Sunybiotech created an independent null allele of elmd-1 via CRISPR-Cas9. 193 The PHX630, elmd-1(syb630) III. mutant contains 1784-bp deletion, where except for exon I, all 194 exons were removed (Supplementary Fig. 2B). elmd-1 (syb630) III. were outcrossed to wild type 195 two times.

Fluorescent transgenes for IFT proteins: GOU2162 che-3(cas443[gfp::che-3]) I; $\quad x b x-$ 198 1(cas502[xbx-1::tagRFP]) V; GOU2362 ift-74(cas499[ift-74::gfp]) II.; EJP76 vuaSi15

199 [pBP36; Posm-6::osm-6::eGFP; cb-unc119(+)] I; unc-119(ed3) III; osm-6(p811) V; 200 N2;lqIs2[osm-6::gfp], N2;ejEx[osm-3::GFP + pRF4]; N2;ejEx[kap-1::gfp+pRF4]; EJP81

201 vuaSi24 [pBP43; Pift-140::ift-140::mCherry; cb-unc-119(+)]II; unc-119(ed3) III; ift202 140(tm3433) V; jhuEx [ift-140::GFP+pRF4]; Ex[rpi-2::GFP+xbx-1::tdTomato+pRF4]; MX76 203 dpy-5(e907); $n x E x(b b s-7: \because g f p+d p y-5(+))$.

204 Fluorescent transgenes for ciliary proteins: PHX1180, wdr-31(syb1180 [wdr205 31(T05A8.5)::GFP]); PHX1328, wdr-31(syb1328[wdr-31(R261-de) $):: G F P])$ (Disease mimicking 
variant); OIK1042 N2;turEx21 [arl-13p::wdr-31 (T05A8.5)::GFP::unc-54 3'UTR +rol-6\} (5 ng);

207 OIK1044 N2;turEx23[elmd-1p::GFP::elmd-1 (C56G7.3)::unc-54 3'UTR +rol-6\} (5 ng);

208 OIK1045 N2;turEx24[arl-13p::GFP::elmd-1 (C56G7.3)::unc-54 3'UTR +rol-6\} (5 ng);

209 OIK1046 N2;turEx25[elmd-1p(C56G7.3)::GFP::unc-54 3'UTR +rol-6\} (50 ng); OIK1047

210 N2;turEx26[wdr-31 (T05A8.5)p::GFP::unc-54 3'UTR +rol-6\} (50 ng); N2;Ex[mks-2::GFP + 211 tram-1::tdTOMATO + pRF4]; MX1409 N2; nxEx785[tax-4:gfp + Posm-5::xbx-1::tdTomato +

212 rol-6(su1006)]; vuaSi21[pBP39; Pmks-6::mks-6::mCherry; cb-unc-119(+)]II; $\quad$ MX2418

213 N2;nxEx1259[pbbs-8::PLC-delta PH::GFP;MKSR-2::tdTomato; coel::GFP]; PY8847 214 oyIs65[str-1p::mcherry]; Ex[str-1p::nphp-4::gfp, unc122p::dsRed]. All PHX strains were 215 generated using CRISPR/Cas9 by Sunybiotech. The list of extensive transgenic and mutant strains 216 was provided in Table S2.

\section{Fluorescence and Confocal microscopy for C. elegans}

219 Fluorescence images (dye assay) and time-lapse movies (IFT movies) were captured using an epi220 fluorescence upright microscope (Leica DM6 B) (3 frames per second, and up to 120 frames). The 221 epi-fluorescence upright microscope was controlled with iQ3.6.2 Andor software and was attached 222 with an Andor iXon Ultra 897 EMCCD (an electron-multiplying charge-coupled device camera). 223

224 The LSM900 confocal microscope with Airyscan 2 (ZEN 3 Blue edition software) was used to 225 capture the high-resolution Z-stack images. On microscope slides, a drop of 2-3\% agarose was 226 used to create an agarose pad, and worms were then moved to the agarose pad containing 1-3 $\mu 1$ 227 of $10 \mathrm{mM}$ levamisole (an anesthetic agent). Images were collected at $0.14 \mathrm{~m}$ intervals with a Plan 
ApoChromat $63 \mathrm{x} / 1.40$ NA target, then analyzed with Blue edition software ZEN 3 to create Zstacks, and processed with ImageJ (NIH) software (Schneider et al., 2012).

\section{In vivo intraflagellar transport assay for IFT frequency}

232 The time-lapse movies of GFP-labelled IFT proteins were analyzed with the automatic kymograph 233 analyzing tools KymographClear and KymographDirect, both of which are ImageJ based 234 (Mangeol et al., 2016). KymographClear was used to produce kymographs from time-lapse 235 movies. We examined each produced kymograph and calculated IFT frequency for IFT-74::GFP 236 (a single copy transgene), OSM-6::GFP (a single copy transgene), OSM-3::GFP, and IFT237 140::GFP in wild type, wdr-31;elmd-1 double and $w d r-31$;elmd-1;rpi-2 triple mutants.

\section{Osmotic Avoidance Assay for assessing cilia functions in C. elegans}

240 Right before each experiment, we freshly prepared an $8 \mathrm{M}$ fructose solution containing 241 bromophenol blue and made a ring of $8 \mathrm{M}$ bromophenol blue solution using $1 \mathrm{ml}$ pipette tips. 1242 day adult worms of wild type and $o s m-5(p 813)$ mutants were always tested as positive and negative 243 controls before evaluating the mutant strains (Haycraft et al., 2001). Wild-type worms are 244 supposed to remain in the ring under normal conditions, while osm-5(p813) mutants may be able 245 to escape due to a defect in the cilia structure. Every assay lasted 5 minutes, and five worms were 246 placed in the ring. At least three separate experiments (on different days) were conducted for each

247 strain, and the total number of worms was more than 40 for wild type and $o s m-5(p 813)$, and more 248 than 80 for the rest of mutants. Fig. S4A was a combined representation of the last two independent 249 experiments. 


\section{Whole-mount in situ hybridization in zebrafish}

252 Whole-mount in situ hybridization was performed as previously described (Thisse et al., 2004).

253 The wdr31 cDNA with $\mathrm{T} 7$ promoter was PCR amplified, with forward primer:

254 ATGGGGAAGCTACAGAGCAAGTTC and primer:

255 TAATACGACTCACTATAGAAGCGAGCCACTTCAGTGATACTG， from a homemade

256 cDNA library of zebrafish embryos at $24 \mathrm{hpf}$. The antisense probe for $w d r 31$ was then transcribed

257 with digoxigenin-labeled UTPs and T7 RNA polymerases (Roche, Basel, Switzerland). The

258 stained embryos were dehydrated in glycerol and photographed with a Nikon SMZ1500

259 stereomicroscope (Nikon, Tokyo, Japan).

261 Mutagenesis of $w d r 31$ in zebrafish using the CRISPR/Cas9 technology

262 Generation of zebrafish mutants using the CRISPR/Cas9 system was carried out as previously

263 described (Chang et al., 2013). Briefly, two gRNAs targeting sequences in $w d r 31$ were chosen as 264 following: 5'- ACCCATGTGTGTTGGGTACC-3' and 5'-GAAGCCATCCAGGAGTTCAG-3'.

265 gRNA templates were PCR amplified and gRNAs were in vitro transcribed with T7 transcriptase 266 (NEB, cat\# M0251S). gRNAs and Cas9 protein (NEB, cat\# M0251S) were simultaneously injected 267 into the embryos at one-cell stage.

\section{Immunofluorescence staining and microscopy}

270 Immunostaining was performed as previously described (Xu et al., 2017). Briefly, embryos were 271 fixed in cold Dent's fixative ( $80 \%$ methanol: $20 \%$ dimethyl sulfoxide) at $-20^{\circ} \mathrm{C}$ overnight and then 272 stored in methanol. Samples were permeabilized with $0.005 \%(\mathrm{~m} / \mathrm{v})$ trypsin for 30 minutes. 273 Samples were blocked with blocking buffer $(10 \%[\mathrm{v} / \mathrm{v}]$ goat serum in PBST), followed by 274 incubation with primary antibody anti-acetylated tubulin (1:2000; Sigma-Aldrich, St. Louis, MO) 
275 and then secondary antibodies conjugated with Alexa Fluor 568 (1:500; Invitrogen, Carlsbad, CA).

276 Samples were mounted on ProLong Gold Antifade Mountant with DAPI (Invitrogen) and images

277 were taken with a Nikon A1R confocal microscope.

278

279 Software and algorithms

280 Zen Blue Zeiss https://www.zeiss.com/corporate/int/home.html

281 Andor iQ3 Andor https://andor.oxinst.com/

282 Fiji $\quad$ ImageJ $\quad \underline{\text { https://fiji.sc }}$

283 ImageJ ImageJ https://imagej.nih.gov/ij/

284 Illustrator (CS5.1) Adobe, USA https://www.adobe.com/

285 KymographClear Peterman Lab https://sites.google.com/site/kymographanalysis

$286 \mathrm{R} \quad$ R Core Team https://www.r-project.org/

288 Statistical analysis and Generating Figures

289 For statistical analysis of dye assay, Fisher's exact test was performed. For data involving

290 continuous variables like IFT speed and cilia length, Mann-Whitney U-test or Welch's t-test was

291 used depending on the distribution of data. All statistical tests were performed using R software.

292 The codes and files needed to generate figures and perform statistical analysis were openly shared, 293 and the files and codes for making figures and performing statistical analysis may be accessed at

294 https://github.com/thekaplanlab/WDR31-ELMOD-RP2.

295 Gateway Cloning (mammalian constructs)

296 Constructs for ELMOD3 (HsCD00288286) and WDR31 (HsCD00045652) were purchased 
297 from Harvard Medical School. LR Reaction (Invitrogen, USA) was performed to transfer the target 298 sequence into destination vectors ((N)RFP, (N)CFP plasmids) with a subsequent transformation 299 into E.coli DH5 $\alpha$. DNA was isolated according to Monarch ${ }^{\circledR}$ Plasmid Miniprep Kit (BioLabs, 300 USA) and the PureYield® Plasmid Midiprep Protocol System (Promega, USA). Verification of 301 successful cloning was done by sequencing (Eurofins, Luxembourg).

\section{Immunofluorescence Staining (Mammals)}

304 hTERT-RPE1 cells were transiently transfected according to Lipofectamine ${ }^{\mathrm{TM}} 3000$ Reagent 305 Protocol with ELMOD3-CFP and WDR31-RFP constructs followed by serum starvation for three 306 days to induce cilia formation. Cells were fixed with $4 \%$ PFA for $45 \mathrm{~min}$ at $4{ }^{\circ} \mathrm{C}$, permeabilized for 307 5min with $0.3 \%$ PBST and blocked with $10 \%$ goat serum in PBST at $4^{\circ} \mathrm{C}$ overnight. A primary 308 antibody for ARL13B (1:50; Proteintech, Germany) and a secondary antibody conjugated to Alexa 309 Fluor 647 (1:350; Invitrogen, USA) were used. Cells were mounted using Fluoromount-G 310 (Invitrogen, USA).

\section{Microscopy Setup (Mammals)}

312 Images were captured using a Leica TCS SP8 scanning microscope (Leica Microsystems IR

$313 \mathrm{GmbH}$, Germany). The setup includes $488 \mathrm{~nm}, 532 \mathrm{~nm}$ and $635 \mathrm{~nm}$ pulsed excitation lasers as 314 well as 100x oil immersion objective lens (NA 1.4) and a hybrid detector (HyD). Pixel number 315 was 1024 x 1024 and optimal pixel size was determined by Nyquist calculation resulting in a size 316 of $43 \times 43 \mathrm{~nm}$ in XY. Additionally, Z-steps should not exceed $131 \mathrm{~nm}$ when acquiring a stack. 317 Laser intensity was adjusted for each sample and images were recorded with $2 x$ frame averaging. 


\section{Image Processing (Mammals)}

321 Acquired images were processed (LasX, Leica Microsystems), deconvoluted (Huygens Software, 322 SVI, Netherlands) and edited (FIJI software). The deconvolution was performed with a Classical 323 Maximum Likelihood Estimation (CMLE) algorithm under experimentally defined settings. The 324 background level was software estimated, the quality threshold was 0.001 , the number of iterations 325 was 50 and signal-to-noise ratio was set to 20. All images were brightness-corrected for the 326 purpose of presentation.

Exome sequencing analysis.

329 Informed written consent was obtained from all participants under an IRB approved protocol 330 (KFSHRC RAC\# 2080006). Whole exome sequencing was performed on the DNA sample 331 collected from proband as previously described (Saudi Mendeliome Group, 2015). Variant 332 classification followed the recommendations of the American College of Medical Genetics 333 (Richards et al., 2015).

\section{Sequence analysis}

335 Sanger sequencing and segregation analysis were done for the proband and family members.

\section{Results}

\section{WDR31 and ELMOD are evolutionarily conserved constituents of cilia}

338 As part of our ongoing effort to reveal novel cilia genes, using single-cell RNA-seq data, we 339 conducted comparative expression analysis in C. elegans and discovered that the WDR31 and 340 ELMOD orthologues, WDR-31 (T05A8.5) and ELMD-1 (C56G7.3), are likely expressed 341 exclusively in ciliated sensory neurons (manuscript in preparation). The head (amphid) and tails 
342 (phasmid) of C. elegans contain a total of 60 ciliated sensory neurons, and the expression patterns

343 of genes can be reliably analyzed using fluorescence tagged promoter markers. Consistent with

344 our predictions, promoter-based GFP reporters revealed that both WDR-31 and ELMD-1 are

345 expressed in most of the ciliated sensory neurons (Fig. S1A). To explore if both proteins are

346 concentrated within cilia, we investigated their subcellular localization in C. elegans and created

347 CRISPR/Cas9-mediated knock-in of WDR-31::GFP and a transgenic animal expressing GFP-

348 tagged ELMD-1. The endogenously expressed WDR-31::GFP is concentrated at the ciliary base

349 in both head and tails, where it colocalizes with the basal body marker $\gamma$-tubulin (TBG-1, the 350 ortholog of human TUBG1), and the IFT-140 (human IFT140) basal body signal (Fig. 1A, B, C,

351 and D), so our co-localization data suggests that WDR-31 is a new cilia-associated protein.

352 ELMD-1 is the sole orthologue of the three human ELMOD proteins (ELMOD1-3) that function 353 as GTPase-activating proteins (GAP). We found that GFP::ELMD-1 localizes to the periciliary 354 membrane compartment (PCMC) and the basal body (BB) (marked with IFT-140), but is proximal 355 to the MKS-6-labeled transition zone that is adjacent to the BB (Fig. 1E, F, and G) (Kaplan et al., 356 2012).

358 To determine if both human WDR31 and ELMOD3 (also known RBED1, the top blastp hit for 359 elmd-1) are concentrated within cilia in mammalian cell lines, we generated hTERT-RPE1 cells 360 co-expressing WDR31-RFP and ELMOD3-CFP and stained them with a ciliary marker ARL13B. 361 Furthermore, WDR31-RFP or ELMOD3-CFP were transiently transfected into hTERT-RPE1 362 cells, and they were additionally stained for ARL13B. Our super-resolution microscopy analysis 363 revealed that both WDR31 and ELMOD3 are enriched in the cilium (Fig. 2A, B and Fig. S2B 364 and C). Taken together, our complementary approach demonstrates that both WDR31 and 365 ELMOD3 are evolutionarily conserved proteins associated with cilia. 
367 WDR31 is a poorly characterized gene encoding a 367 amino acid long conserved protein with 368 four WD domains, but with our WDR31 and ELMOD3 localization data, WDR31 and ELMOD3 369 emerge as a strong gene candidate for ciliopathy (Fig. 3A). Our ciliopathy patient database was scanned for the presence of mutations in WDR31 and ELMOD3, and we identified a homozygous nonsense variant (NM_001012361.2: c.802C >T; p.Arg268*, rs ID: rs764141336) in a ciliopathy male patient. The conversion of the arginine residue at position 268 to a stop codon (R268*) in

373 WDR31 prevents the translation of the last 99 amino acids with a WD domain (Fig. 3A). This 374 variant is very rare in gnomAD (MAF 0.0000239) and in our database of 2,379 ethnically matched exomes (MAF 0.001). The patient possesses renal agenesis, congenital hydrocephalus, right 376 microtia, right preaxial polydactyly, and congenital heart disease (Fig. 3B, C D, and E). Sanger 377 sequencing confirmed the existence of a homozygous nonsense variant in the affected male while 378 parents and two unaffected sisters (II:3, II:5) are heterozygotes for the mutation and unaffected 379 brother and sister (II:4) had the wildtype genotype (Fig. 3F, G). Taken together, our clinical data 380 linked a novel cilia gene WDR31 to a typical ciliopathy.

\section{Wdr31 regulates ciliogenesis in the Zebrafish ear}

383 We turned our interest into zebrafish to develop an in vivo model for WDR31 to examine the role 384 of WDR31. We first used the whole-mount in situ hybridization (ISH) within zebrafish to visualize 385 the expression pattern of $w d r 31$ during zebrafish embryo development up to 1-day post386 fertilization. The dynamic and wide expression of $w d r 31$ during zebrafish embryonic development 387 (2 cell and 8 somite stages (SS)) has become restricted to the otic vesicle and brain region at 24 388 hours post-fertilization (24 hpf) (Fig. 4A). We next knocked out (KO) wdr31 in zebrafish with the 389 CRISPR/Cas9 system and the embryos showed heart edema and otolith malformation. As wdr31 
is expressed in the otic vesicle and the otolith development requires cilia, we checked the cilia in 391 the otic vesicle. The cilia bundle in the lateral crista (LC) of the otic vesicle was stained with 392 acetylated tubulin in wild type and $w d r 31 \mathrm{KO}$, and although we found that the length of LC cilia 393 was comparable with wild type, the width of the cilia bundle was reduced by $33 \%$ in LC of the 394 otic vesicle compared to that in the control embryos, suggesting the cilia number is decreased (Fig. 395 4B, C, and D). Our zebrafish work indicates that WDR31 plays a role in ciliogenesis in the 396 zebrafish ear.

\section{Functional redundancy of WDR31, ELMOD, and RP2 for determining cilia morphology}

We next wanted to gain mechanistic insight into the functions of $w d r-31$ in cilia biogenesis. To

this end, we employed the nematode C. elegans and obtained/generated three $w d r-31$ alleles: tm10423 (160-bp frameshift causing deletion), syb1568 (1888-bp deletion removing all exons except exon I), and tur003 (1276-bp deletion removing a large portion of exon II and exon III, IV, and exon V) (Fig. S3B). In C. elegans, the lipophilic fluorescent dye-uptake assay is employed to 404 indirectly evaluate the cilia structure, and our analysis revealed $w d r-31$ mutants display wild type 405 level dye-uptake in both head (amphid) and tail (phasmid), suggesting cilia structures are likely unaffected in these mutants (Fig. 4E). To address whether loss of $w d r-31$ leads to subtle defects

407 in cilia morphology, we expressed the fluorescence-based marker str-1pro:mCherry that labels the 408 AWB cilia in $w d r-31$ mutants. The AWB dendritic tip extends the fork-like cilia in the wild type, 409 and our confocal microscopy analysis showed that the structure and length of AWB cilia are 410 comparable with that of wild type AWB cilia, suggesting, contrary to the importance of WDR31

411 for ciliogenesis in the zebrafish ear, the loss of $w d r-31$ alone does not result in a severe defect in 412 cilia structure (Fig. 4B, D, and F). The lack of an apparent cilia phenotype in C. elegans may be 
413 attributed to a functional redundancy for WDR-31 in cilia biogenesis, and functional redundancy

414 is indeed a common phenomenon in ciliopathy-related genes. For example, while the absence of 415 individual ciliopathy genes encoding MKS/MKSR proteins or NPHP proteins (NPHP-1, NPHP416 4) does not result in serious cilia defect, the loss of $n p h p-4$ (the ortholog of human NPHP4) in 417 combination with $m k s-6$ (the ortholog of human CC2D2A) causes more severe cilia related defects 418 (transition zone membrane association defects) (Williams et al., 2011). We next explored genetic 419 interaction between $w d r-31$ and elmd-1, since our results showed that both protein products are at 420 the ciliary base (Fig. 1C, D, E, and F). We discovered that the wdr-31(tm10423); elmd-1(syb603) 421 double mutant (hereinafter referred to as "double mutant") has a partial Dyf defect in both head 422 and tail neurons, which was rescued by expression of a transgene containing the wild type elmd-1 423 sequence (Fig. 4E and Fig. S3C, $p<0.0001$, Fisher's exact test). Using the AWB cilia 424 fluorescence marker, we subsequently set out to visualize the ciliary structures in the double 425 mutant and single mutants. The AWB cilia in the double mutants have an extra projection in the 426 middle part of the cilia, and the cilia are of normal length (7\% (N: 100) and $52 \%(\mathrm{~N}: 134)$ of extra 427 projections in WT and double mutant, respectively) (Fig. 4F and G, and Fig. S4B, C and D).

428 We explored genetic interaction between $w d r-31$, elmd-1, and rpi-2 (the X-linked retinitis 429 pigmentosa protein RP2 and a GTPase-activating protein) since ELMD-1 and WDR-31 430 localizations are reminiscent of RPI-2 localisation in C. elegans (Fig. S5B) (Williams et al., 2011). 431 We, therefore, first generated an elmd-1;rpi-2 double mutant, which revealed no Dyf phenotype. 432 However, when we created wdr-31;elmd-1;rpi-2 triple mutants (hereinafter referred to as "triple 433 mutant"), we observed severe synthetic Dyf phenotype, which is significantly rescued by the 434 introduction of a wild-type copy of $w d r-31$ or elmd-1 or rpi-2 (Fig. $\mathbf{4 E}$, Fig. S3C, D, p $<0.0001$, 435 Fisher's exact test). 
436 We then sought to examine AWB cilia morphology in triple mutants and compared it with the 437 wdr-31;elmd-1 and rpi-2;elmd-1 double mutants. The Dyf defect of triple mutants was indeed 438 accompanied by significant changes in the AWB cilia that displayed the ectopic projections, 439 including a backward projection from the base of AWB cilia in all three independent $w d r-31$ alleles 440 and as wells as ectopic projections from the middle part of AWB cilia ( $0 \%$ backward projection in

441 wild type vs over $30 \%$ backward projection in triple mutants; $\mathrm{p}<0.0001$, Fisher's exact test), but 442 the length of AWB cilia (long) in triple mutants was similar to that of wild type (Fig. 4F, F, and 443 Fig. S4B, C, D).

445 Finally, we investigated the effect of $w d r-31, r p i-2$ and elmd-1 loss on osmotic avoidance 446 behaviour which is mediated by sensory cilia. Our analysis showed that double and triple mutants 447 display defective osmotic avoidance (Osm) behaviour, suggesting cilia are not functioning 448 properly (Fig. S4A).

Loss of WDR-31 and ELMD-1 leads to the ciliary accumulations of OSM-3/KIF17 Kinesin motor and Complex B components

452 We next aimed to investigate the mechanism by which WDR-31, ELMD-1, and RPI-2 regulate 453 cilia morphology in C. elegans. To this end, we investigated whether these genes affect the 454 localization of IFT proteins and IFT motors, including kinesin motors (Kinesin II and OSM4553 /KIF 17), dyneins, and IFT components (IFT-A and IFT-B). The IFT complex and motor proteins 456 are critical for cilia construction and maintenance because they deliver ciliary constituents from 457 the cell body to the cilia. In C. elegans, two kinesin motors Kinesin II and OSM-3/KIF17 458 (heterotrimeric kinesin-II and homodimeric OSM-3) work cooperatively to carry the IFT complex 459 in the middle segment of the channel cilia in an anterograde direction, while OSM-3/KIF17 
transports the entire IFT complex (IFT-A, IFT-B, and BBSome) in the distal part of channel cilia.

461 We generated single, double, and triple mutants expressing fluorescence tagged IFT and motor 462 proteins. Using single-copy transgenes, we found that IFT-B components accumulate at cilia tips 463 and/or middle of cilia in double mutants (96\% ciliary tip accumulations for IFT-74/IFT74, N: 26 464 and $82 \%$ ciliary tip accumulations for OSM-6/IFT52, N: 46) but the localization of GFP::CHE-3 465 (human dynein heavy chain DYNC2H1) remains less affected in double mutants (27\% minor 466 middle cilia accumulations for CHE-3; N: 48, see supplementary Movie S1) (Fig. 5A, C and D 467 and Supplementary Movie S1, 2, 4). Furthermore, we next investigated the localization of 468 GFP::CHE-3 in triple mutants because the cilia morphology of triple mutants is more severe than 469 that of $w d r-31 ;$ elmd-1 or $w d r-31, r p i-2$ or elmd-1;rpi-2 double mutants (Fig. 5F). Compared with 470 wild type and double mutants, we noticed additional IFT abnormalities in two independent triple 471 mutants, including dim cilia staining with GFP::CHE-3 and accumulations of GFP::CHE-3 in the 472 middle/distal part of cilia (81\% middle cilia accumulations, $\mathrm{N}: 96 ; 6.6 \%$ ciliary tip accumulations, 473 N: 90) (Fig. 5A and Supplementary Movie S1). Motor protein OSM-3/KIF17 accumulation 474 within the ciliary tips was detected in double and triple mutants. The size of OSM-3 ciliary tip 475 accumulations appeared to be larger in triple mutants than in double mutants (Fig. 5B, and 476 Supplementary Movie S3). Furthermore, in triple mutants, the dim distal cilia staining was 477 observed for IFT-140::GFP (IFT-A component) (63\% dim distal cilia, N: 47) and XBX$478 \quad$ 1::mCherry (a dynein light intermediate chain) (Fig. 5E and F and Supplementary Movie S5), 479 but the localization and motility of KAP-1::GFP (Kinesin II) remain unaffected (Fig. 5G). Our 480 fluorescence microscopy analysis suggests defects in the return of IFT and OSM-3/KIF17 kinesin 481 motors from the cilia in double and triple mutants. We hypothesized that certain IFT particles 482 would be unable to return from the ciliary tips due to potential defects in the IFT returning, so we 483 measured IFT particle frequency in wild type and affected mutants to explore if the number of IFT 
484 particles entering into cilia is affected and/or if the return of IFT particles from the ciliary tips is 485 altered. Using time-lapse video coupled with kymography, for a subset of IFT components, we 486 discovered a substantial reduction in the number of IFT particles traveling along the cilia in both 487 directions in double and triple mutants (Fig. 6A, and B). In wild type, the average of IFT-74 488 particles moving in anterograde and retrograde directions ( 33 seconds and $7.5 \mu \mathrm{m}$ ) is 19 and 17 , 489 respectively, while in triple mutants, the anterograde and retrograde IFT-74 particles are 9 and 3. 490 Comparable reductions were observed for IFT-74::GFP (a single copy transgene) in wdr-31;eldm$491 \quad 1$ (the anterograde and retrograde: 19 and 17 in wild type vs 12 and 7 in $w d r-31 ; e l d m-1 ; \mathrm{p}<0.0001$; 492 the Mann-Whitney U test). However, for OSM-6::GFP, the retrograde IFT particles statistically 493 differ between wild type and triple mutants (the retrograde: 19 in wild type, 13 in double mutants; 494 and 11 in triple mutants; $\mathrm{p}<0.0001$; the Mann-Whitney U test) (Fig. 6B). Furthermore, we found 495 significant decline in the IFT transport for OSM-3::GFP in double and triple mutants in both 496 anterograde and retrograde directions, but the decrease in IFT transport was more severe for triple 497 mutants (the anterograde and retrograde: 23 and 20 in wild type; 16 and 11 in double mutants; 11 498 and 8 in triple mutants, $\mathrm{p}<0.0001$; the Mann-Whitney $\mathrm{U}$ test) (Fig. 6B). On the other hand, the 499 flux of cytoplasmic dynein motor protein CHE-3 along the cilium was unchanged in both 500 directions in both double and triple mutants, while FT-140 transport declined in both directions 501 (Fig. 6B). Our findings suggest that with the exception of heterotrimeric Kinesin II, all IFT proteins 502 studied, including dynein CHE-3, OSM-3 kinesin, IFT-B, and IFT-A components, display certain 503 defects in $w d r-31$ related mutants, either ciliary accumulations for dynein CHE-3, OSM-3 and 504 IFT-B component or dim distal cilia staining for IFT-A component and dynein motors (CHE-3 505 and XBX-1). 


\section{The ciliopathy associated variant in WDR-31 displays cilia defects in C. elegans}

509 Like human WDR31, C. elegans WDR-31 has WD-repeat domains (Fig. 3A, and Fig. S2A). In

510 the ciliopathy patient, we found the arginine-to-a termination codon substitution at residue 268 of

511 human WDR31 that removes the 99 amino acids containing a WD domain, but the functional

512 significance of this change is currently unknown in the context of cilia biogenesis. To investigate

513 whether the last 99 amino acids in WDR-31 is essential for the subcellular localization of WDR-

51431 to the cilia base and the function of WDR-31, the CRISPR/Cas9 was used to engineer the $C$.

515 elegans model harboring the corresponding WDR31 deletion (named WDR-31 ${ }^{\text {R261-del) }}$ (Fig. 7A).

516 Using confocal microscopy, we determined the subcellular localization of WDR-31 ${ }^{\text {R261-del }}$, which

517 revealed that, similar to wild type WDR-31::GFP (Cas9/CRISPR GFP knock-in), WDR-31 R261-

518 del::GFP is situated at the base of cilia proximal to the MKS-6 marked transition zone (Fig. 7B,

519 and Fig. S5A), suggesting that the last WD repeat within WDR-31 are not necessary for the ciliary

520 base localization. In contrast, we found several cilia abnormalities when the $w d r-31^{\text {R261-del }}$ variant

521 was combined with elmd-1 and rpi-2. First, similar to $w d r-31 ;$ elmd-1;rpi-2 triple mutants, the triple

$522 w d r-31^{R 261-d e l} ;$ elmd-1;rpi-2 mutants exhibit a synthetic Dyf phenotype, suggesting structural

523 defects in cilia in this triple mutant (Fig. 4E, 7C). Consistent with this expectation, we find that

524 the AWB cilia structural abnormalities in $w d r-31^{\text {R261-del }}$;elmd-1;rpi-2, including backward

525 projections from the cilia base, were comparable to three separate $w d r-31$ mutants (Fig. 4G, 7D,

526 E, over 30\% backward projection in triple mutants; $p<0.0001$, Fisher's exact test). As expected,

527 we observe no Dyf phenotype and cilia structural defects in $w d r-31^{R 261-d e l}$ single mutants (Fig. 7C,

528 D).

529 Finally, because WDR-31, ELMD-1, and RPI-2 localize at the base of cilia, we wanted to examine 530 the role of these proteins in the ciliary gate, and we chose the TRAM-1 protein (the ortholog of 531 human TRAM1), which surrounds the PCMC in C. elegans but stays outside of cilia (Williams et 
al., 2011). Our confocal microscopy analysis revealed that the TRAM-1 protein enters into cilia in

533 all examined $w d r$-31 triple mutants, including $w d r-31^{\text {R261-del }}$;elmd-1;rpi-2 but not in $w d r-31^{\text {R261-del }}$

534 single mutants (Fig. 7E). Taken together, all these data from C. elegans indicates the human R268*

535 mutation (an equivalent of R261-del in C. elegans) in WDR31 observed in the ciliopathy patient

536 is likely a pathogenic variant.

WDR-31 and ELMD-1 are required for recruitment of BBS-7, a BBSome component, to cilia

539 To find out the underlying reason for why IFT-B components and OSM-3/KIF17 specifically

540 accumulate at the ciliary tip in double and triple mutants, we hypothesized that the IFT complex

541 destabilization might be due to defects in the BBSome assembly. The IFT-B components were

542 previously reported to accumulate at the ciliary tip in BBSome mutants (Blacque, 2004; Wei et al.,

543 2012; $\mathrm{Xu}$ et al., 2015). We found that ciliary tip accumulations of IFT-74:GFP, an IFT-B

544 component, in $b b s-8(n x 77)$ mutants were comparably similar to that of double and triple mutants

545 (Fig. 8A). We next explored the localization of BBS-7::GFP, a core member of the BBSome, in

546 double and triple mutants. BBS-7::GFP is lost or significantly diminished in the cilia of double

547 and triple mutants, but BBS-7::GFP localization persists at the ciliary base in these mutants (Fig.

548 8B). The time-lapse movie analysis of BBS-7::GFP together with kymography reveals that BBS-

$5497::$ GFP movements were undetectable in $27 \%$ and $42 \%$ of cilia in the head in double and triple

550 mutants, respectively (Supplementary Movie S6). We were able to quantify the frequency of

551 BBS-7::GFP even though the density of trajectories (IFT particles) on kymographs was weak in

552 double and triple mutants relative to wild type (Fig. 8C). Our analysis revealed a substantial

553 decrease in the average of BBS-7::GFP particles translocating in the anterograde and retrograde

554 directions in the remaining worms that display IFT (average anterograde and retrograde IFT 
particles: 18 and 19 for wild type; 10 and 11 for double mutant; 2 and 2 for triple mutant, $\mathrm{p}<$ 0.0001; the Mann-Whitney U test) (Fig. 8C, D and Supplementary Movie S6). We next explored

557 the localization of transition fiber protein FBF-1 and TAX-4 (a membrane protein) in the triple 558 mutants because FBF-1 was implicated in the ciliary entry of IFT machinery (Wei et al., 2013). 559 Our data reveals that the localization of these proteins remains unaffected in the triple mutants

560 (Fig. 5SB and D). Taken together, our results suggest that because the BBSome complex is unable 561 to gain access to cilia in double and triple mutants, IFT-B components and OSM-3/KIF17 likely 562 accumulate excessively in the distal portion of the cilia while IFT Complex A component staining 563 the distal segment cilia become dim (Fig. 8E).

\section{Discussion}

\section{WDR31 is a new ciliopathy gene required for cilia biogenesis}

567 The list of both the primary and motile ciliopathy genes has been increasingly expanding with the 568 large-scale adoption of next-generation sequencing technologies in the exploration of genetic 569 causes for these diseases. So far, over 190 ciliopathy genes have been discovered (Wheway et al., 570 2019). Despite substantial advances in identifying many ciliopathy genes, elucidating the

571 underlying genetic mutations and their functions in ciliopathies represent a major challenge. In the 572 current work, we identified WDR31 as a novel cilia protein and relate it to ciliopathy. We found a 573 truncating variant in WDR31 in a family of nine, with the only affected child harboring a 574 homozygous truncating variant. The patient displayed ciliopathy-related clinical features, 575 including congenital hydrocephalus, polydactyly, and congenital heart disease. The human 576 counterpart variant results in an abnormal cilia structure in C. elegans, confirming the conclusion 577 that this variant likely disrupts WDR-31 functions. 
579 Human WDR31 is a member of the WD40-repeat protein (WDR) family, and it was previously 580 understudied even though several members of the WD40-repeat protein family, including WDR34, 581 WDR35, and WDR60, have been linked to cilia (Blacque et al., 2006; Patel-King et al., 2013; 582 Rompolas et al., 2007). Strikingly, we found that WDR31 is a ciliary protein, and knocking it out 583 in zebrafish using CRISPR/Cas9 causes ciliary defects, including a decrease in the number of cilia

584 in the LC. Furthermore, the link between WDR31 and cilia was significantly strengthened by 585 findings from C. elegans. First, the expression of the WDR31 orthologue (WDR-31) is restricted 586 to the ciliated sensory neurons, and WDR-31 localizes to the base of cilia. The exclusive cilia 587 expression pattern of $w d r-31$ in C. elegans is similar to that of ciliary and ciliopathy genes like 588 ARL-13/ALR13b, IFT, and BBS (Blacque et al., 2005). Second, CRISPR/Cas9 mediated knock 589 out of WDR-31 combination with the ELMOD orthologue (ELMD-1) and RP2 orthologue (retinitis 590 pigmentosa 2; RPI-2) results in an abnormality in cilia functions and structure in C. elegans. Our 591 findings reveal that WDR31 is a new ciliopathy protein required for cilia morphology in both 592 zebrafish and C. elegans, suggesting the evolutionarily conserved role of WDR31 in cilia biology.

594 Functional redundancy between WDR-31, ELMD-1, and RPI-2

595 Our genetic analysis in C. elegans provides evidence for partial functional redundancy for $w d r-31$, 596 elmd-1, and rpi-2 in regulating cilia morphology and the trafficking of kinesin-IFT-BBSome 597 complexes. Though cilia morphology is disrupted in $w d r-31 ;$;elmd-1;rpi-2 triple mutants, with 598 altered recruitment of kinesin, IFT, and BBS proteins, simultaneous elimination of elmd-1 and $r p i-$ 5992 did not result in severe defects in cilia and IFT. Our interpretation for these data is that the 600 additive defects in the triple mutants were likely due to $w d r-31$, and WDR-31 is a central player 601 in controlling cilia morphology and IFT machinery. However, we cannot rule out the possibility 602 of microtubule defects in cilia ultrastructure in elmd-1;rpi-2 double mutants. Additional work is 
603 needed, and a transmission electron microscope (TEM) may be used to reveal further details in the 604 ultrastructures of cilia in these double mutants.

606 How do these proteins work together to regulate cilia-related phenotypes? While WDR31 is a 607 poorly characterized gene, the roles of human RP2 and ELMOD proteins in cilia biology are better 608 understood. Human RP2 localizes to the cilium and the basal body and displays GAP activity 609 toward two ARF family members, ARL2 and ARL3, both of which were linked to cilia biology 610 (Evans et al., 2010; Schwarz et al., 2017; Wright et al., 2011), while the ELMOD protein family 611 (ELMOD1-3) has GAP activity for ARL2 (ELMOD1 and ELMOD3) and ARF6 (ELMOD1)

612 (Ivanova et al., 2014; Jaworek et al., 2013; Johnson et al., 2012; Miryounesi et al., 2019). 613 ELMOD2 localizes to the basal body, and our study showed ELMOD3 localizes to cilium (Turn 614 et al., 2021). Taking into account the fact that RPI-2 and ELMD-1 are GAPs, and loss of these two 615 GAP proteins likely result in the overactivation of their target G proteins, how does WDR-31 616 function with these GAP proteins? One possibility is that WDR31 may function downstream of 617 regulatory GTPases, including ARL2, ARL3, or unidentified GTPases, activated by these GAP 618 proteins. One downside of this explanation is that $w d r-31$ single mutants did not have significant 619 ciliary and IFT defects; but, if this were valid, we would expect to observe further anomalies in 620 cilia and IFT in $w d r-31$ single mutants, close to the removal of these three genes. Alternatively, 621 the activity/function of overactive GTPases can be somehow regulated by WDR-31, maybe WDR62231 may have a GAP activity for the regulatory GTPases or it may have a GTPase activity. While 623 WDR-31 does not seem to have a GAP domain, HHMER search revealed that human WDR31 has 624 a distant sequence resemblance to a nucleoside-triphosphatase (NTPase) domain (NACHT) 625 containing protein in Penicillium camemberti (Gabler et al., 2020). PSI-BLAST search confirmed 626 this result (unpublished data). 
628 Furthermore, the ciliary roles of these two GAP proteins might be independent of their GAP 629 activities, and consistent with this idea, the recent study showed the ciliary roles of ELMOD2 are 630 partially independent of its GAP activity (Turn et al., 2021). However, a variety of evidence 631 suggests that WDR31 and ELMOD3 do not form a complex. First, mass spectrometry-based 632 proteomic analysis for either WDR31 or ELMOD3 showed that neither WDR31 nor ELMOD3 633 contains each other or RP2 (unpublished data). Second, the proper localization of any of these

634 three proteins was independent of each other (unpublished data). Further studies are needed to 635 understand a mechanistic link between WDR31, ELMOD, and RP2, and figure out the independent 636 contributions of WDR31 and both GAP proteins in cilia biology.

\section{How does the WDR31-ELMOD-RP2 regulate the IFT trafficking in cilia?}

639 Based on our results, which involve a decrease in moving IFT particles in anterograde transport, 640 ciliary tip aggregation of OSM-3/KIF17 and IFT-B, and docking of BBSome to the IFT machinery,

641 we propose that these defects likely stem from the failure of BBSome to associate with moving

642 IFT in cilia in double and triple mutants. Consistent with our proposal, the almost comparable

643 IFT/BBSome phenotypes were reported with hypomorphic mutations in $d y f-2$ (WDR19

644 orthologue, an IFT-A component) and $b b s-1$ (a BBSome component), where they showed that 645 while BBSome remains at the base of cilia and does not undergo IFT, the failure of IFT-B 646 reassociation with IFT-A at the ciliary tips results in IFT-B ciliary tip accumulations, despite the

647 fact the association of IFT-A and IFT-B components in anterograde transport persists (Wei et al., 648 2012). They proposed that the BBSome controls the IFT assembly at the ciliary base and IFT 649 turnover at the ciliary tip. However, the IFT defects in the triple mutant are unlikely to be due to 650 mislocalization of transition fiber proteins such as DYF-19 (the FBF1 orthologue), which was 
651 implicated in regulating the ciliary entry of IFT and BBSome, since our data showed that the 652 localization of DYF-19 was not impaired in the triple mutants (Wei et al., 2013). On the other 653 hand, the effect on IFT might be direct since RP2 was previously proposed to control the 654 intraflagellar transport protein IFT20 pool in the peri-basal body and trafficking of Kif17 and Kif7 655 to the ciliary tip (Schwarz et al., 2017). However, several interesting questions have yet to be 656 resolved: what is the mechanism by which WDR-31-ELMD-1-RPI-2 regulates the ciliary entry of 657 the BBSome entry? How does WDR-31-ELMD-1-RPI-2 interact with proteins, including ARL6, 658 that regulate the BBSome recruitment (Jin et al., 2010)? Future study research will help us to 659 understand how these proteins regulate BBSome/IFT trafficking. In summary, this study provides 660 a mechanistic explanation of the function of WDR-31, a new ciliopathy protein, in the regulation 661 of cilia biogenesis and also contributes to our knowledge of the molecular etiology of WDR31 662 mediated disease.

664 Acknowledgments

665 We thank the family of the patient, who participated in the study. We thank Oliver Blacque, Piali 666 Sengupta, Micheal Leroux, the National BioResource Project (NBRP) in Japan, and the CGC in 667 the United States, which is financed by the NIH Office of Research Infrastructure Programs (P40 668 OD010440) for sharing valuable strains. We thank Oliver Blacque and Samuel Katz for critical 669 reading of the manuscript. We thank the Abdullah Gul University Scientific Research Project

670 Coordination Unit (Project number: TOA-2018-110) for providing the funding that initiated the 671 project. M.U. was supported by the Tistou \&amp; Charlotte Kerstan Stiftung. The Leica laser 672 scanning microscope was funded by a grant from Deutsche Forschungsgemeinschaft (INST $673 \quad 2388 / 62-1)$. 

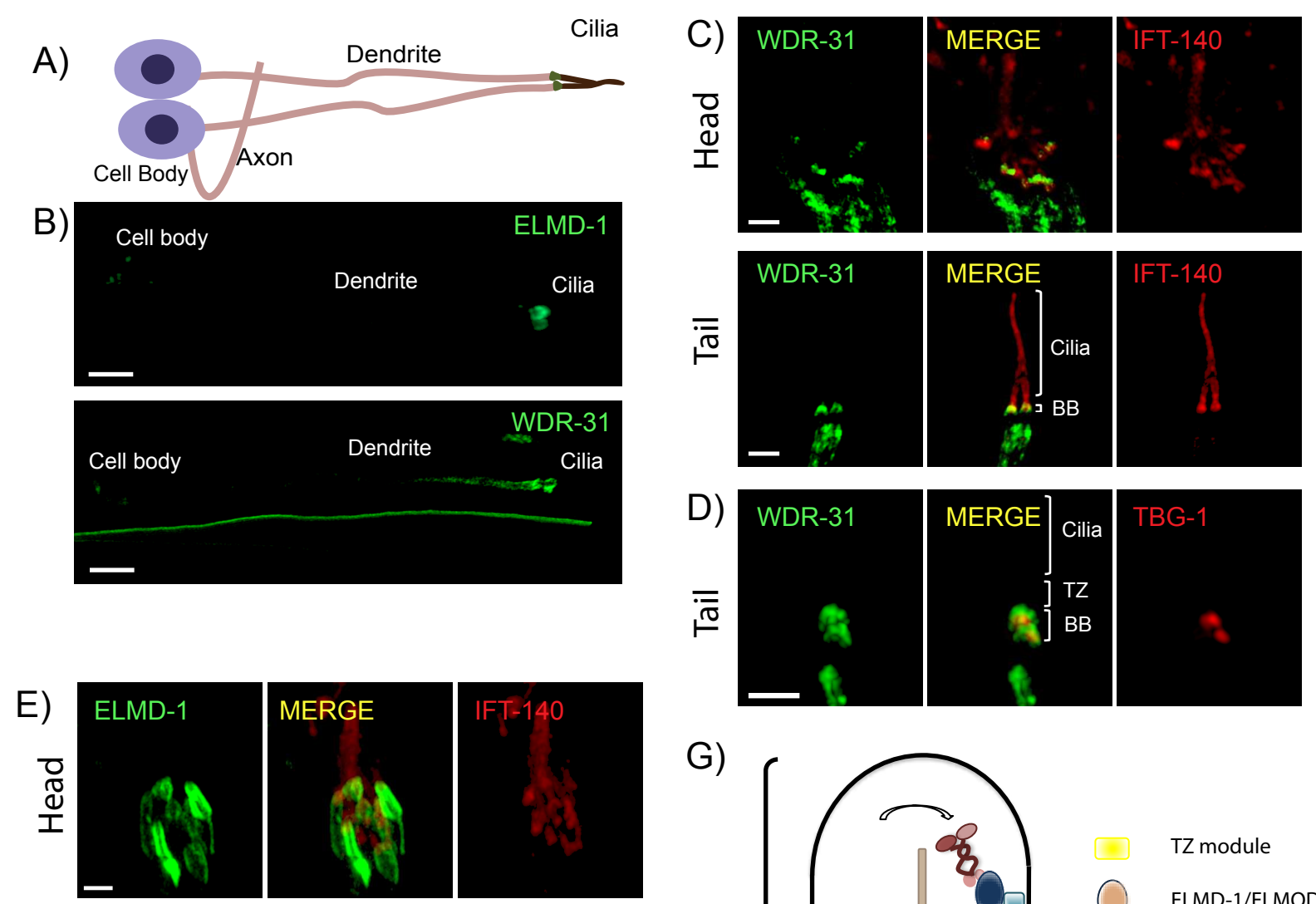

G)
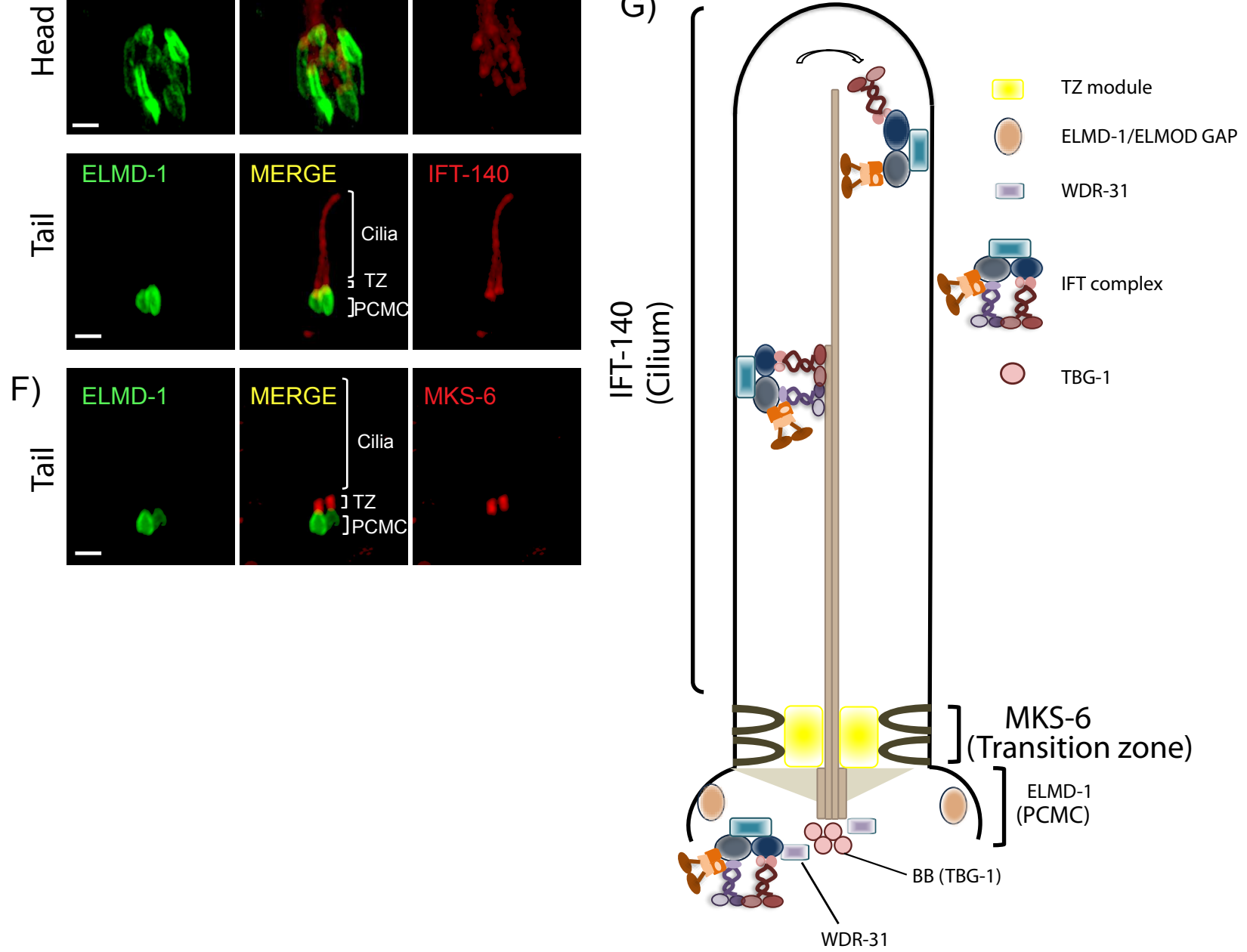

676 Figure 1: WDR-31/WDR31 and ELMD-1/ELMOD proteins are evolutionary conserved 
677 ciliary proteins A, B) Shown are the representative drawing of the PHA/PHB sensory neuron 678 (phasmid neurons located in the tail). Cilia, dendrite, axon and cell soma (cell body) are depicted 679 in the drawing. Fluorescence images from the transgenic strain carrying WDR-31::GFP or 680 GFP::ELMD-1 were displayed in the PHA/PHB sensory neurons. Scale bars: $3 \mu \mathrm{m}$ C, D) Co681 localization of WDR-31::GFP (Green) with the IFT-140::mCherry (Red, an IFT-A component, a 682 ciliary marker) or TBG-1:mKate (Red, $\gamma$-Tubulin, the basal body) in the tail (phasmids) and head 683 (amphid) sensory neurons. TZ and BB denote the transition zone and the basal body, respectively 684 E, F) GFP::ELMD-1 (Green) localizes to the BB and PCMC (the periciliary membrane 685 compartment) proximal to the transition zone. Co-labelling of GFP::ELMD-1 with MKS686 6:mCherry marker (transition zone) or IFT-140::mCherry in the tail (phasmids) and head (amphid) 687 neurons. Scale bars: $2 \mu \mathrm{m} \mathrm{G)}$ 

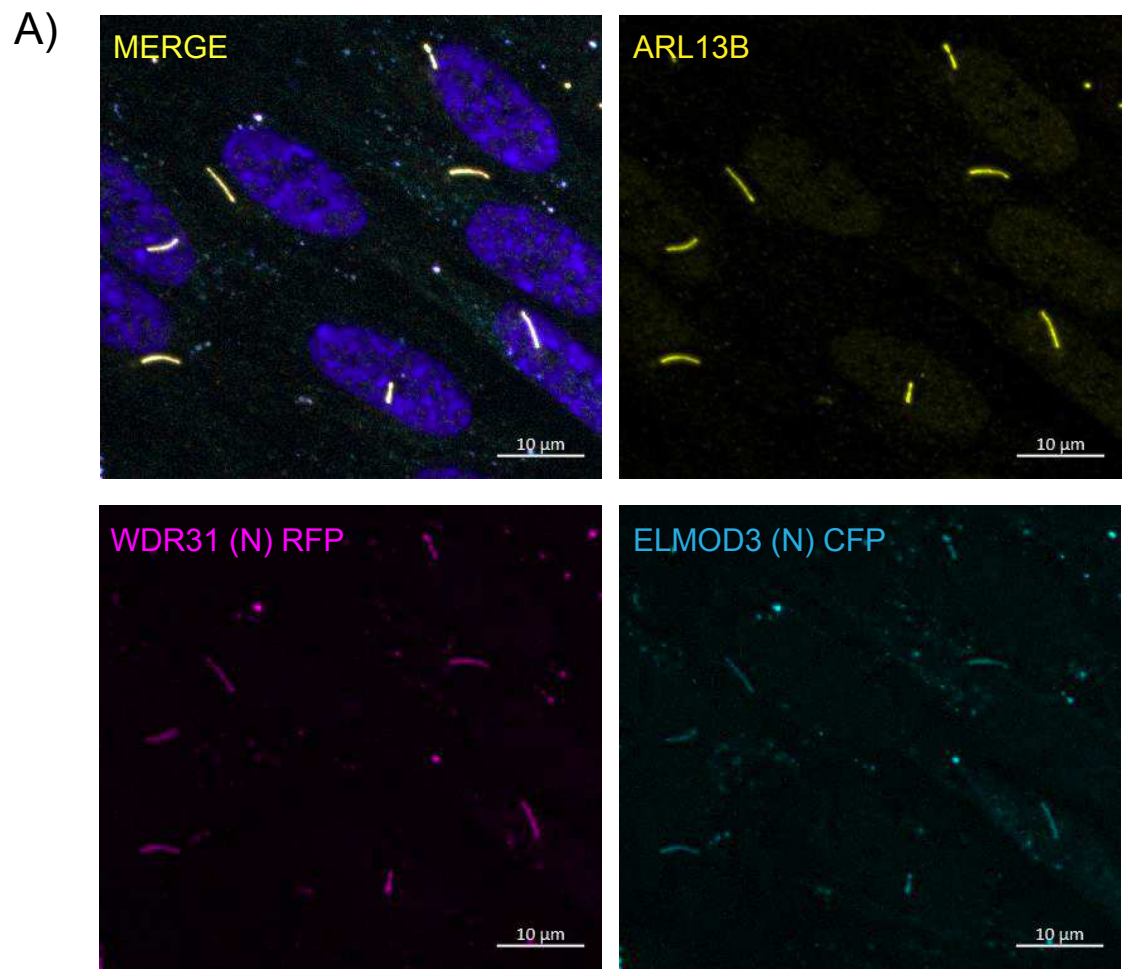

B)
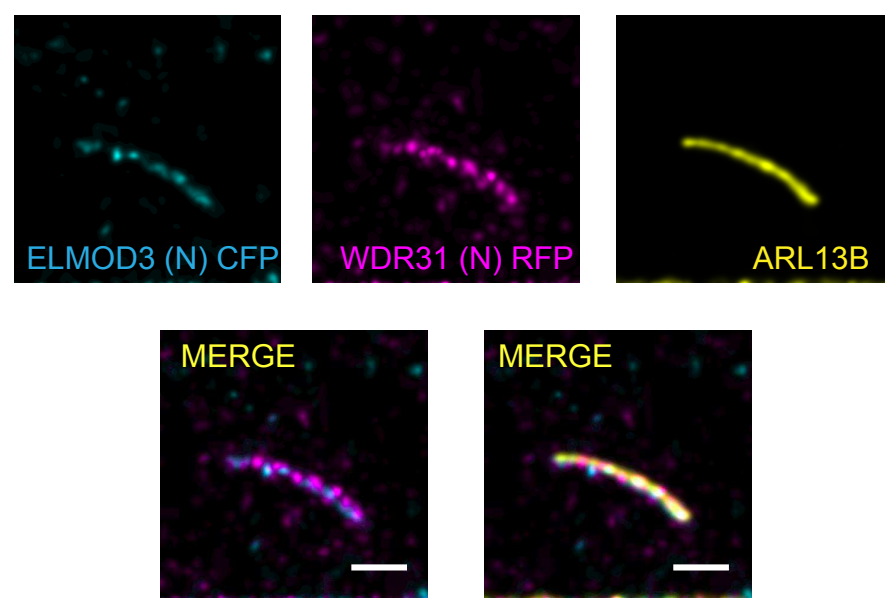

690 Figure 2: ELMOD3 and WDR31 localize to the primary cilium. A, B) Shown are the staining

691 of WDR31 (tagged with cyan fluorescent protein) and ELMOD3 (tagged with red fluorescent

692 protein) together with a ciliary marker ARL13B and DAPI (nucleus) in hTERT-RPE1 cells.

693 hTERT-RPE1 cells were transiently transfected with $100 \mathrm{ng}$ of WDR31:CFP and ELMOD3:RFP.

694 Scale bars: $10 \mu \mathrm{m}(\mathrm{A})$ and $3 \mu \mathrm{m}$ (B) 


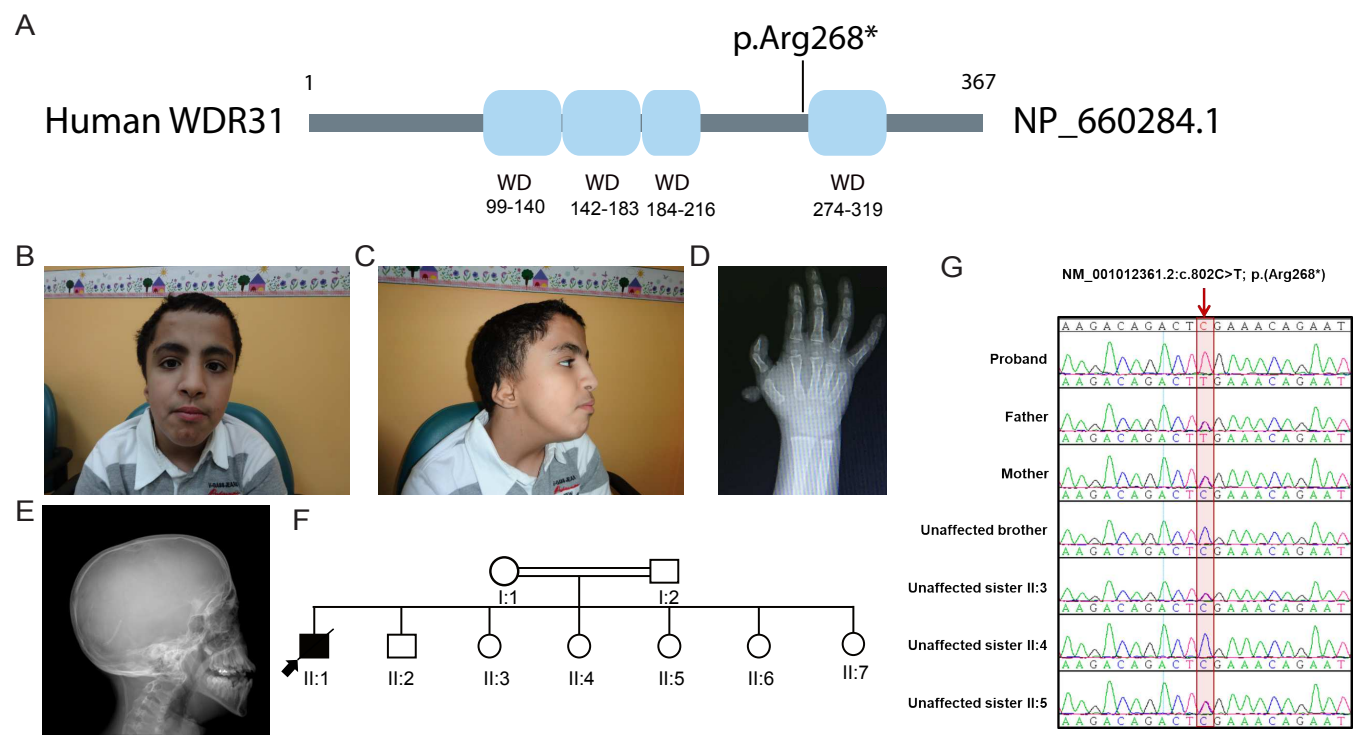

696 Figure 3: A homozygous p.R268* variant in WDR31 leads to a ciliopathy A) Shown is the 697 schematic of WDR31 (NP_660284.1), with 367 amino acids in length and WD domains as tandem 698 repeats. The position of disease associated p.R268* (rs ID: rs764141336) variant is depicted in 699 human WDR31, resulting in a 268 amino acid protein in length. B and C) Clinical images and 700 molecular analysis of the proband. Facial images of the proband (14DG0977) with hydrocephalus 701 and right microtia, D and E) X-ray imaging highlighting right preaxial polydactyly, and 702 hydrocephaly, respectively. F) schematic representation of the pedigree showing consanguinity 703 between the parents (1st degree cousins). The proband is marked with an arrow. G) Sequence 704 chromatogram confirming the variant segregation in the index (homozygote c.802C $>\mathrm{T}$; 705 p.(Arg268*)). The parents and two unaffected sisters (II:3, II:5) are carriers (heterozygotes), and 706 the unaffected brother and sister (II:4) are wild types. 


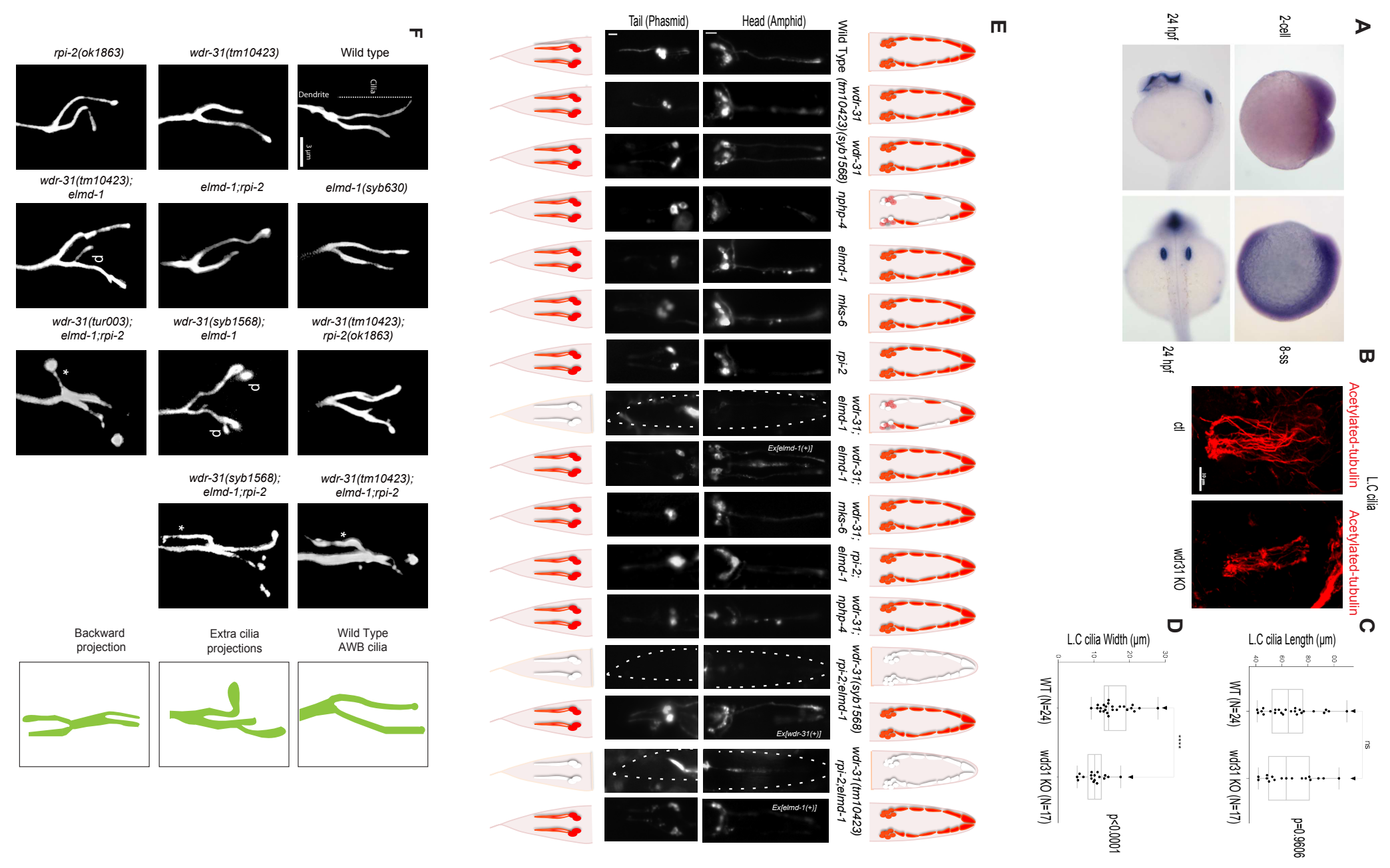


708 Figure 4: WDR31 regulates ciliogenesis in zebrafish A) Shown are the expression pattern 709 analysis of WDR31 in zebrafish embryos. Wdr31 is ubiquitously expressed before the 710 segmentation stage ( 2 cell and 8 somite stages (SS). The expression of $W d r 31$ becomes limited to 711 the otic vesicle and brain region at 24 hours post fertilization ( $24 \mathrm{hpf}$ ). Shown are cilia of lateral 712 crista (LC) of the otic vesicle, stained with acetylated-tubulin, in wild type and $W d r 31$ knockout,

713 generated via CRISPR/Cas9. C and D) The length of cilia in the lateral crista (LC) of the otic 714 vesicle remains unaffected in zebrafish $W d r 31$ knockout while the cilia number is decreased, as 715 shown with the measurement of width of cilia lateral crista (LC) of the otic vesicle. E) Shown are 716 the schematic representations of lipophilic fluorescent dye uptake in the head (amphid) and tail 717 (phasmid) sensory neurons in C. elegans. Red labelling indicates the normal dye uptake while the 718 failure of Dye uptake was shown in white. Fluorescence images show the dye update in the head 719 and tail neurons in the wild type and indicated mutant strains. No dye uptake was observed in both 720 head (amphid) and tail (phasmid) of $w d r-31(t m 10423) ; e l m d-1$ double mutants, $w d r-$ 721 31(tm 10423);elmd-1;rpi-2 and wdr-31(syb1568);elmd-1;rpi-2 triple mutants. Ex[elmd-1(+)] 722 rescues the dye uptake defects of $w d r-31$ (tm10423);elmd-1 double and $w d r-31($ tm 10423);elmd723 1;rpi-2 triple mutants, while $E x[w d r-31(+)]$ rescues the Dye uptake defects of $w d r-$ 724 31(syb1568);elmd-1;rpi-2 triple mutants. Scale bars: $10 \mu \mathrm{m}$. F) Fluorescence images show the 725 morphology of AWB cilia (fork-like structure located in the head) in wild type and indicated 726 mutant backgrounds. The backward projection from the cilia is shown with asterisks $(*)$ while $p$ 727 indicates the ectopic projections from the middle parts of cilia. The AWB cilia in wild type and 728 affected mutants are shown in schematic sketches. 


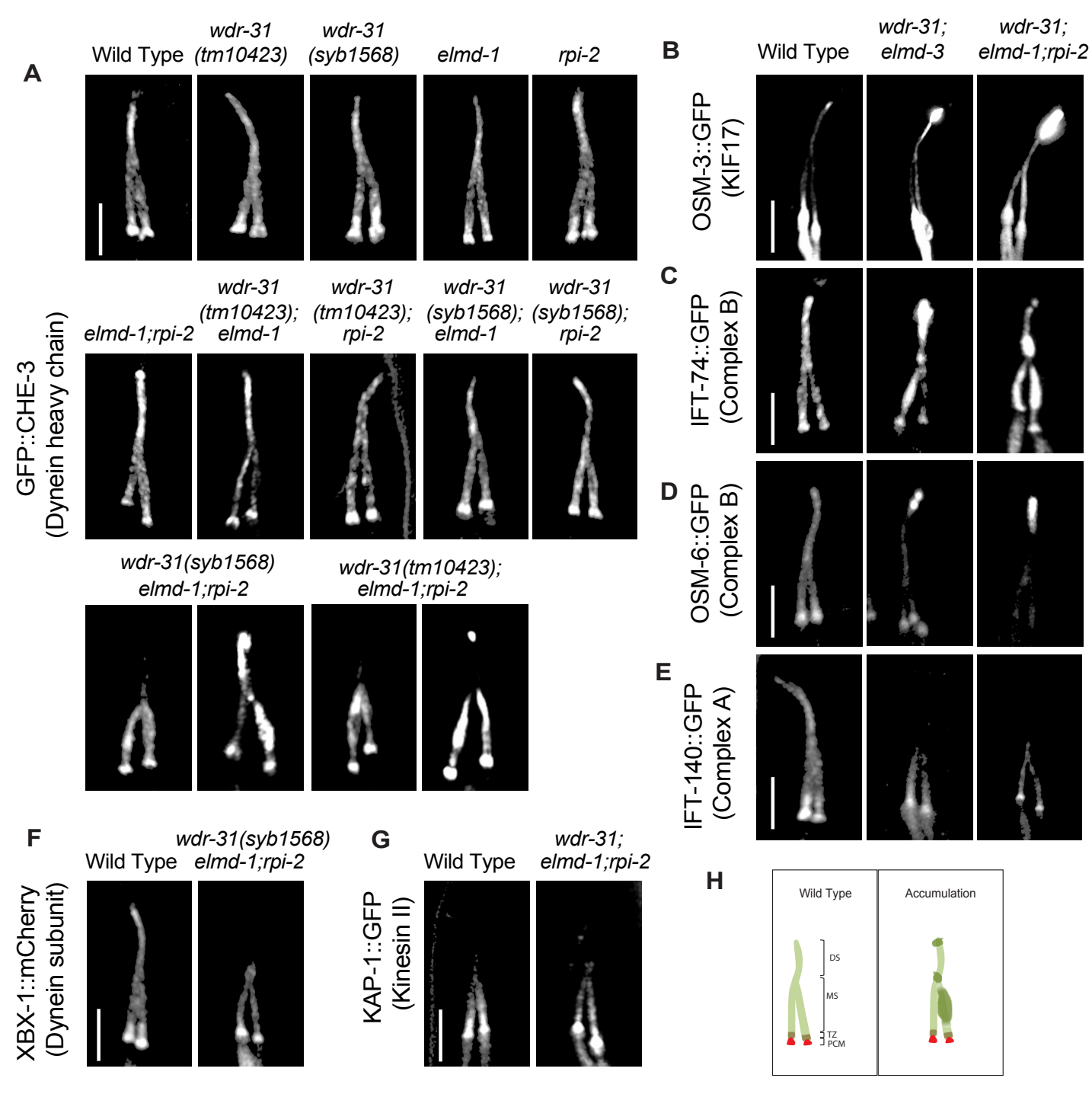

730

731 Figure 5: IFT-B proteins and OSM-3/KIF17 accumulate at the ciliary tip. Shown are

732 fluorescent images of PHA/PHB cilia (phasmid tail). A) Fluorescent images from a single copy

733 GFP::CHE-3 (human dynein heavy chain DYNC2H1) in wild type and indicated mutant

734 backgrounds are displayed. GFP::CHE-3 accumulations within cilia and dim distal cilia staining

735 were observed in two distinct triple mutants (wdr-31(tm10423);elmd-1;rpi-2 and wdr-

736 31(syb1568);elmd-1;rpi-2). Scale bars: $3 \mu \mathrm{m} \mathrm{B,} \mathrm{C,} \mathrm{D,} \mathrm{E,} \mathrm{F)} \mathrm{Confocal} \mathrm{microscopy} \mathrm{analysis} \mathrm{of}$

737 IFT-A (IFT-140::GFP) and IFT-B complex components (OSM-6/IFT52::GFP and IFT-74::GFP)

738 revealed differential abnormalities in the transport of IFT-A and IFT-B components in double 
739 (wdr-31;elmd-1) and triple mutants. The localization of XBX-1::mCherry (Dynein subunit) in $740 w d r-31(s y b 1568) ;$ elmd-1;rpi-2 triple mutants phenocopies the dim distal cilia staining of IFT-A 741 (IFT-140::GFP) in the $\mathrm{w} d r$-31(tm10423);elmd-1;rpi-2 triple mutants. OSM-3/KIF17 Kinesin 742 motor accumulates at the ciliary tips in $\mathrm{w} d r$-31(tm10423);elmd-1 double and $\mathrm{w} d r$ 743 31(tm 10423);elmd-1;rpi-2 triple mutants. Compared to double mutants, the ciliary tip staining is

744 more strong in the triple mutants. Scale bars: $3 \mu \mathrm{m} \mathrm{G)} \mathrm{Fluorescent} \mathrm{images} \mathrm{from} \mathrm{Kinesin} \mathrm{II} \mathrm{motor}$ 745 (KAP-1::GFP) revealed that the restricted middle segment localization of KAP-1 remains 746 unchanged in $\mathrm{w} d r$-31 (tm 10423);elmd-1;rpi-2 triple mutants. Scale bars: $3 \mu \mathrm{m}$ H) Shown are the 747 drawings of phasmid cilia (PHA/PHB sensory neurons in the tail) in wild type and mutants 748 showing ciliary accumulations. 

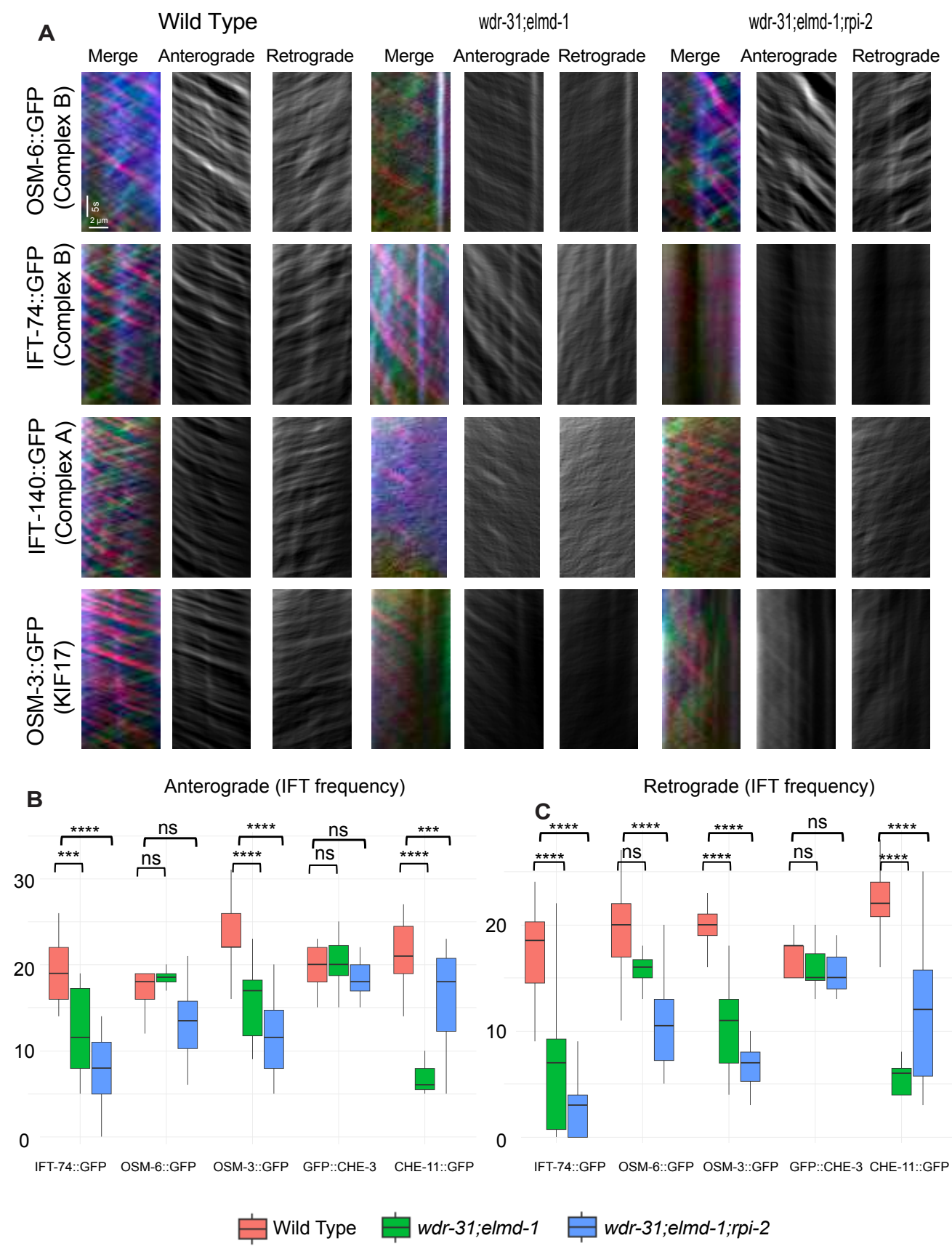

750 Figure 6: Measurement of anterograde and retrograde IFT transport frequency A) Shown

751 are representative kymographs of GFP tagged IFT proteins translocating in the tail cilia (PHA/PHB

752 sensory neurons) of wild type and indicated mutants. Kymographs for anterograde, retrogrades

753 and merged (Red \& Green) were generated with ImageJ equipped with KymographClear. The

754 trajectory represents a moving IFT particle, and the average number of moving IFT particles in 
755 wild type and indicated double and triple mutants was calculated by counting all trajectories in 756 each kymograph. Travel time and distance are shown on kymographs. B) Box-and-Whisker charts 757 with error bars were created to visualize the average number of IFT anterograde and retrograde 758 particles between wild type and indicated mutants. The Mann-Whitney U test was used to measure 759 statistical analysis and significance. The four and three asterisks $(* * * *$ and $* * *)$ at the top of the 760 brackets indicate that the $\mathrm{p}$ value between the two strains is less than 0.0001 and 0.001 , 761 respectively, suggesting statistical significance. Ns stands for "not significant." 
A

Human SCSNGFGGE GCEATLWDLRQTRNRI CEYKGHFQTVA 282 NP_001012361.1

Chimp SCSNGFGGE GCEATLWDLRQTRNR I CEYKGHFQTVA 281 XP_003312284.1

Macaca SCSNGFGGE GCEATLWDLRQTRNR I CEYKGHFQTVA 282 XP_001102129.1

Rat SCSSGFGGE GCEATLWDLRQTRNR I CEYKGHFQT VA 282 NP_001011976.2

C. elegans SCSNGFNNDGCETSIYDVRNPRQS-REARGHEGNVT 274 NP_494344.1

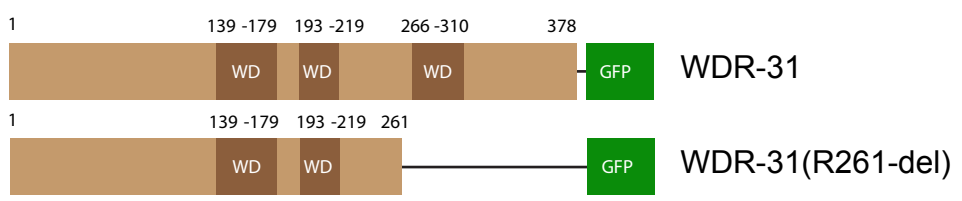

B

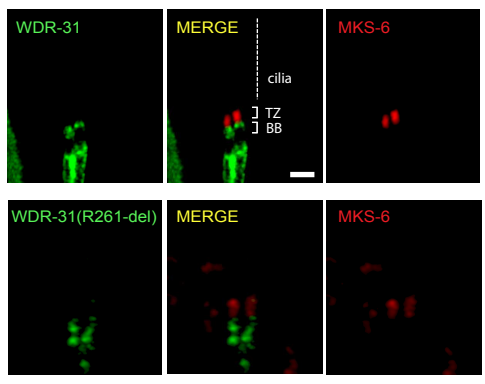

C

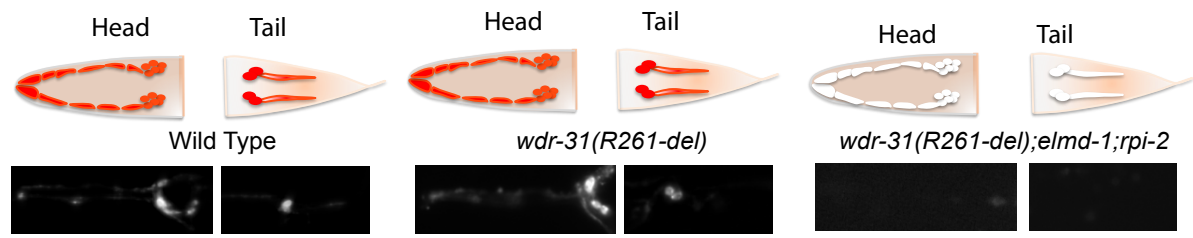

D

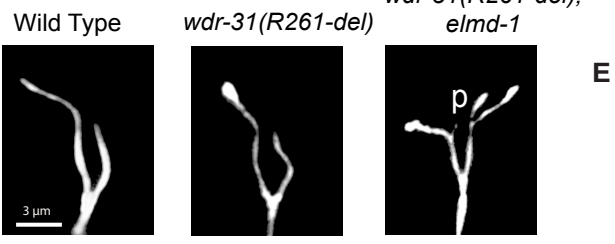

$w d r-31(R 261-d e l) \quad w d r-31(t m 10423) ; w d r-31(s y b 1568)$; elmd-1;rpi-2 elmd-1;rpi-2

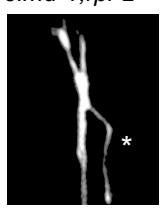

$w d r-31$ (tur003) elmd-1;rpi-2
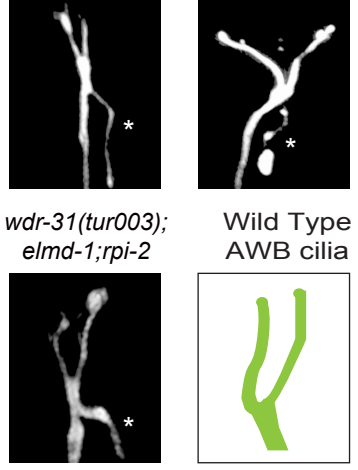

Wild Type AWB cilia

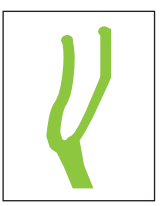
elmd-1,rpi-2

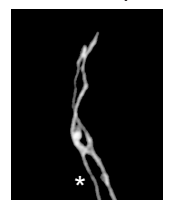

Backward

projection

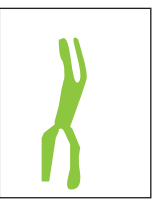

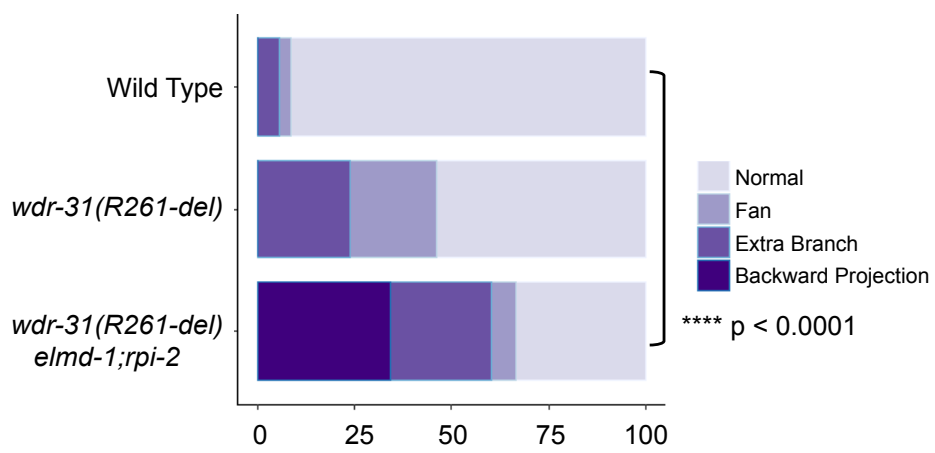
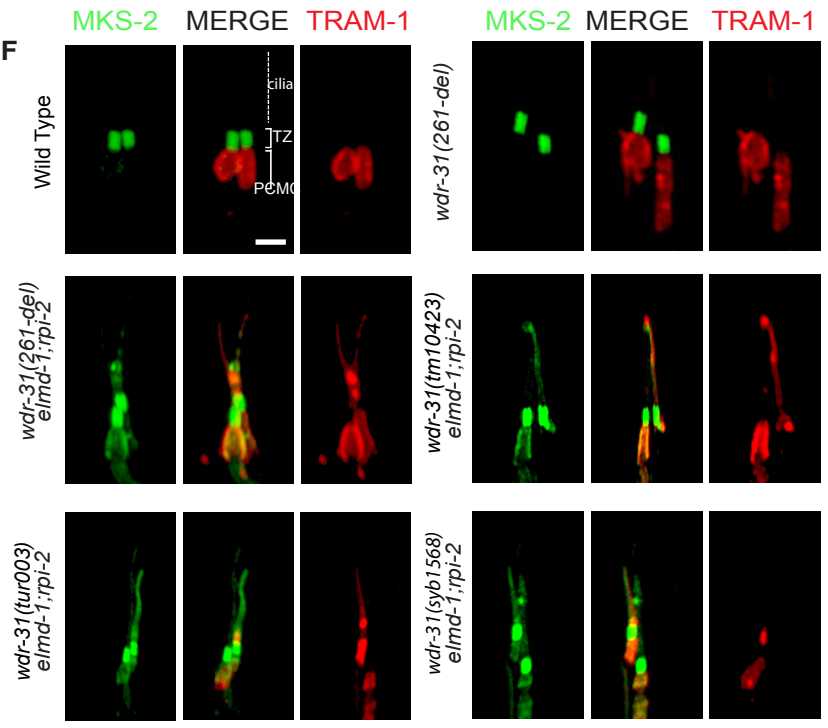

variant phenocopies the cilia related anomalies in the null wdr-31 C. elegans mutants. A)

765 Shown are multiple sequence alignments of WDR31 between human, chimp, macaca, rat and $C$. 766 elegans. The arginine residue at 268 was converted to a stop codon (R268*) in the ciliopathy 767 patient, causing the last almost 100 amino acids not to be translated, and the arginine residue at 768268 of human WDR31 was conserved between humans and other organisms included. To mimic 769 a comparable human patient variant in C. elegans, the CRISPR/Cas9 was used to create the 770 deletion starting from the arginine residue at 261 of C. elegans WDR-31. B) Shown are 771 fluorescence images of WDR-31::GFP and WDR-31( ${ }^{\text {R261-del }):: G F P ~ w i t h ~ t h e ~ t r a n s i t i o n ~ z o n e ~ m a r k e r ~}$

772 MKS-6:mCherry in the tail. Both WDR-31 markers are located directly underneath the TZ. C)

773 Shown are fluorescence images together with cartoon representations. $w d r-31\left(^{\text {R261-del }) ; e l m d-1 ; r p i-}\right.$

7742 triple mutants exhibit dye-filling (Dyf) phenotype. D) Fluorescence images and the

775 representative cartons display the fork-like structure of the AWB cilia in wild type and indicated 776 mutant backgrounds. In all examined $w d r-31$ triple mutants, including $w d r-31\left(^{\text {R261-del }) ; e l m d-1 ; r p i-}\right.$

777 2, the backward projections from the base of AWB cilia were observed. The ectopic projection

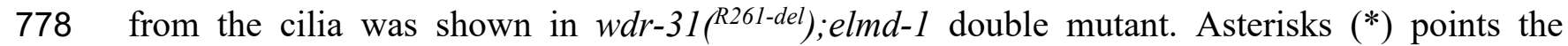
779 backward projection while $\mathrm{p}$ indicates ectopic projections from the middle parts of cilia. E) The 780 percentage distribution of AWB cilia morphology in wild type and indicated mutants is displayed 781 in the graph. F) Confocal fluorescent images exhibit the localization of tdTomato tagged TRAM7821 (a PCMC marker) and MSK-2::GFP (a TZ marker) in wild type and indicated mutants. TRAM7831 leaks into cilia in all four $w d r-31$ triple mutants, including the disease phenocopying mutant. 784 Cilia, PCMC, and TZ are depicted in the fluorescent image. Scale bars: $2 \mu \mathrm{m}$ 785 


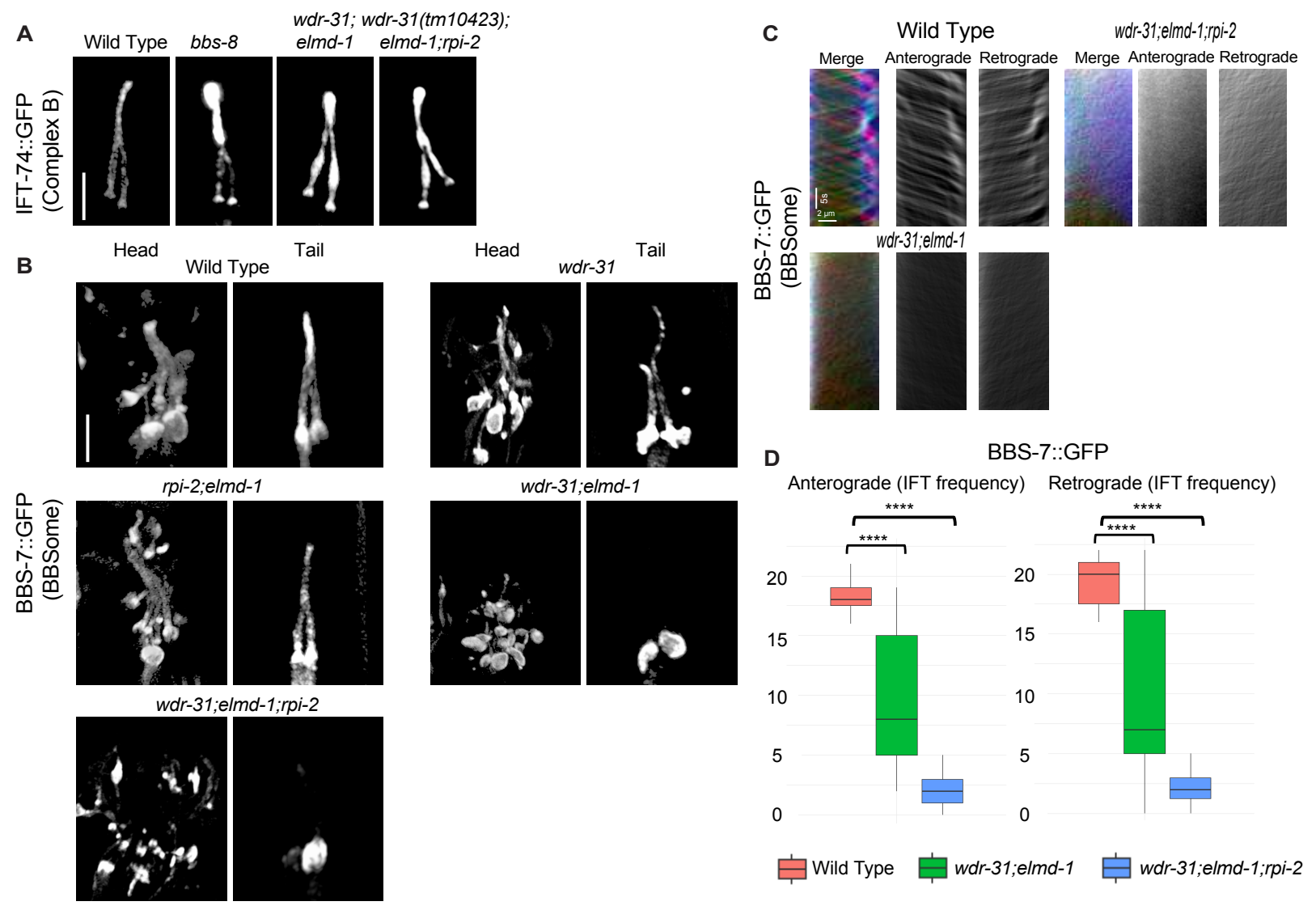

E
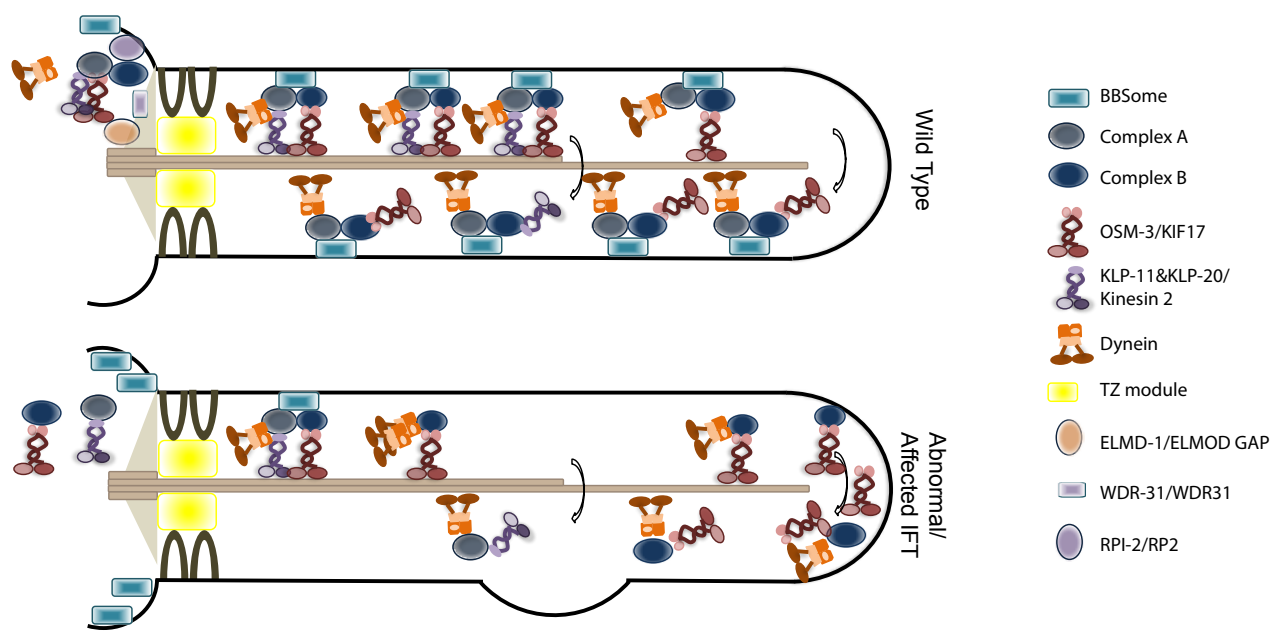

787 Figure 8: WDR-31 and ELMD-1 regulate the recruitment of BBSome to cilia.

788 A) Shown are fluorescence images from the transgenic strain carrying IFT-74::GFP, an IFT-B

789 component, in wild type, $w d r-31$;elmd-1 double mutants, $w d r-31$;elmd-1;rpi-2 triple mutants, and

$790 \quad b b s-8(n x 77)$. The IFT-B subunit IFT-74:GFP accumulates at the ciliary tips and cilia in the tail of 
791 all three mutants. B) Confocal fluorescence images showing the localization of BBS-7:GFP, a

792 BBSome subunit, in the heads and tails of wild type and $w d r-31($ tm 10423); wdr-31;elmd-1; rpi-

793 2;elmd-1 double and wdr-31;elmd-1;rpi-2 triple mutants. Fluorescence images showed absent or

794 weak cilia staining of BBS-7::GFP in both the head and tails of wdr-31;elmd-1 and wdr-31;elmd-

795 1;rpi-2 triple mutants. C) Kymographs were created from time-lapse BBS-7::GFP movies

796 (PHA/PHB cilia) using KymographClear integrated into ImageJ. Shown are representative

797 kymographs for BBS-7::GFP translocating in wild type and indicated mutants. Each trajectory in

798 kymographs was counted. Travel time and distance are included on kymograph D) The graph

799 depicts the average number of BBS-7::GFP particles traveling around cilia in both directions for

800 wild type and indicated mutants. The Mann-Whitney U test revealed statistical significance

801 between the compared strains and that the $\mathrm{p}$ value was less than 0.0001 shown by the four asterisks

$802(* * * *)$ at the top of the brackets E) In wild type, the assembly of the Kinesin-IFT-BBSome 803 complex (Kinesin-II and OSM-3, IFT-B, IFT-A and BBSome) happens at the base of cilia. In the 804 middle segment of amphid and phasmid cilia in C. elegans, both heterotrimeric Kinesin II and 805 homodimeric OSM-3 transport the IFT-A- IFT-B- BBSome complex in an anterograde direction.

806 Heterotrimeric Kinesin II returns to the ciliary base when it reaches the tip of the middle segment 807 of amphid and phasmid cilia, whereas homodimeric OSM-3 is responsible for the anterograde 808 translocation of the IFT-BBSome complex in the distal segment of amphid and phasmid cilia.

809 When the OSM-3-IFT-BBSome complex reaches the ciliary tip, cytoplasmic dynein transports 810 them back to the ciliary base. In $w d r-31 ;$ elmd-1 double $w d r-31 ;$ elmd-1;rpi-2 triple mutants, the 811 BBSome failed to enter into cilia, thus leading to accumulations of OSM-3 and IFT-B components 812 in the ciliary tips. 
A)

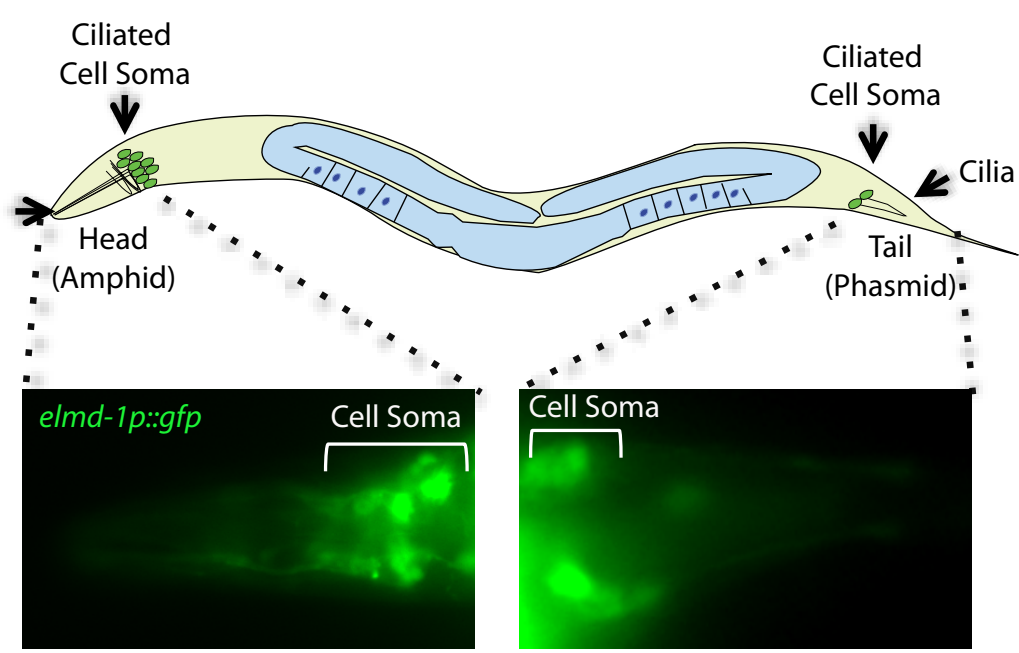

814
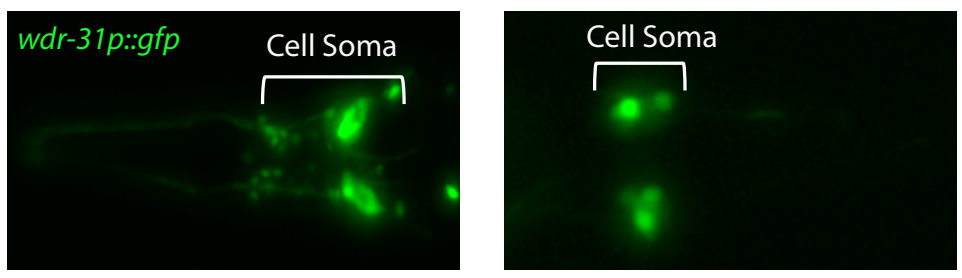

815 Supplementary Figure 1: Expression patterns of WDR-31 and ELMD-1. A) A total of 60

816 sensory neurons are distributed in the head (amphid) and tails (phasmid) of C. elegans. The ciliated

817 sensory neurons are displayed in the schematic of C. elegans. The expression of elmd-

818 1promoter::gfp $(1000 \mathrm{~kb})$ and $w d r-3$ lpromoter::gfp $(1000 \mathrm{~kb})$ were shown in the fluorescence

819 images. The cell of ciliated sensory neurons (cell soma) in the head and tails were displayed in 820 brackets. 

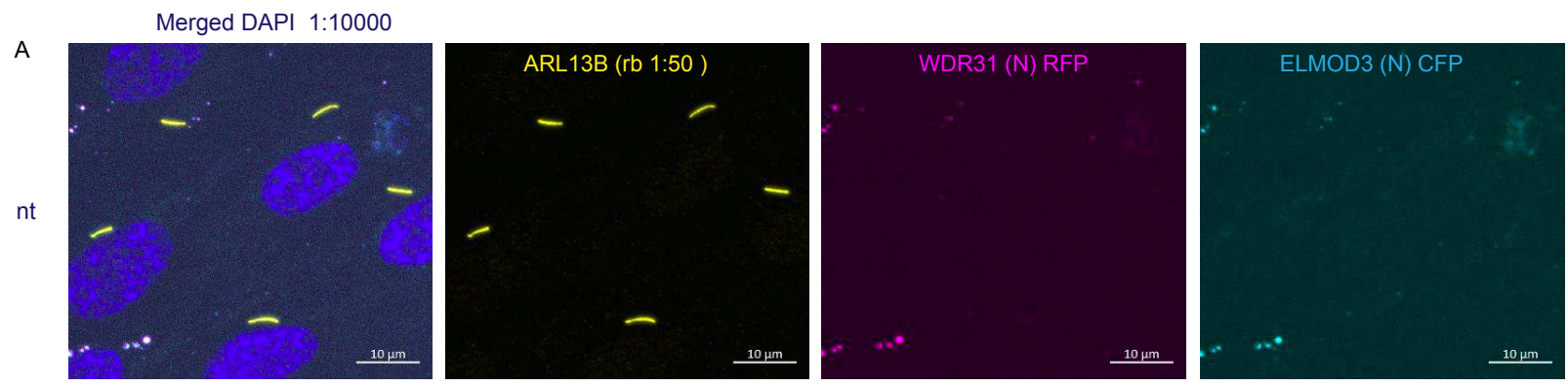

B

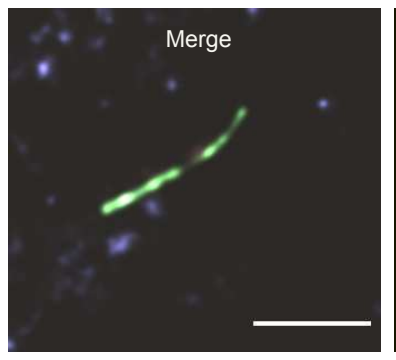

C

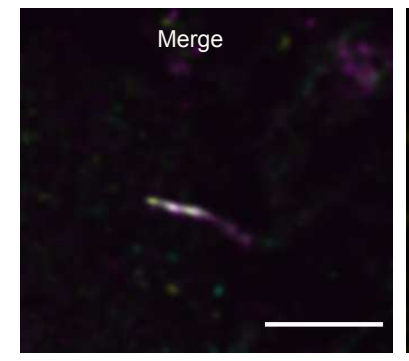

ARL13B (rb 1:50)
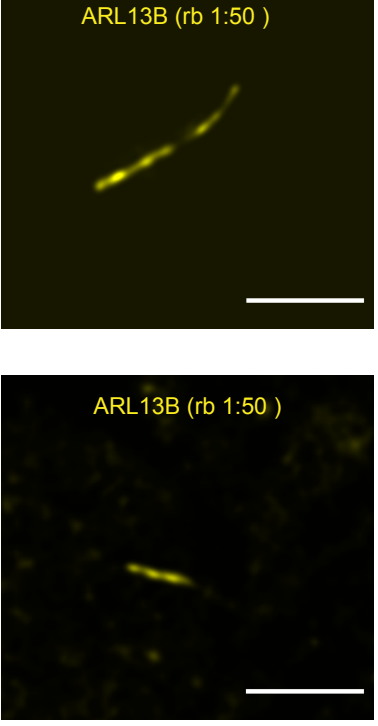
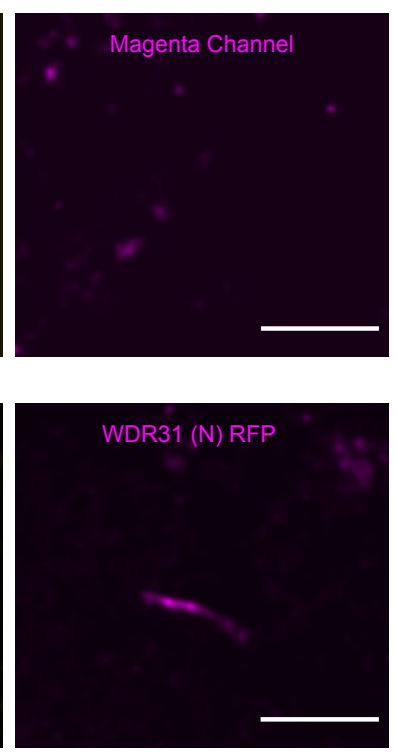
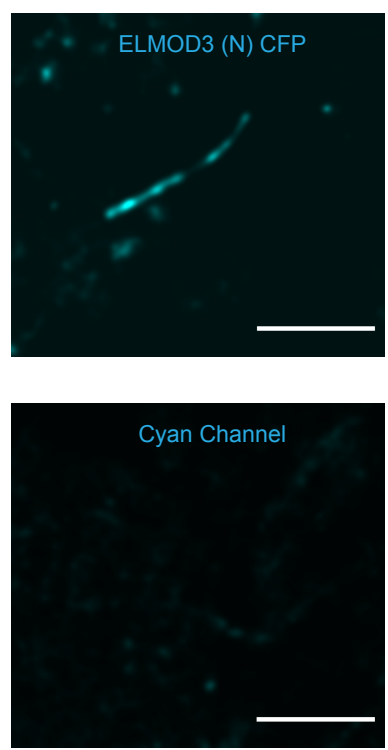

Supplementary Figure 2: Ciliary localization of WDR31 and ELMOD3 in hTERT-RPE1

823 cells A) Images showing immunostaining of a ciliary marker ARL13B and DAPI (nucleus) in

824 hTERT-RPE1 cells. NT represents no transfection of hTERT-RPE1 with WDR31 (tagged with

B) Images showing immunostaining of hTERT-RPE1 cells transfected with ELMOD3-CFP (cyan)

827 and also stained for ARL13B (yellow). The magenta channel only shows some background but no

828 localization to the cilium. Green regions in the "merge" picture show co-localization of both

829 ELMOD3 and ARL13B proteins. Scale bars: $3 \mu \mathrm{m}$. C) Fluorescence images displaying hTERT-

830 RPE1 Fluorescence images of immunostaining for ARL13B (yellow) and WDR31-RFP (magenta)

831 in hTERT-RPE1 cells transfected with WDR31-RFP (cyan). The cyan channel shows some 
832 background, with no cilium localisation. WDR31 and ARL1B proteins are co-localized in the pink 833 sections of the "merge" image. Scale bars: $3 \mu \mathrm{m}$. 


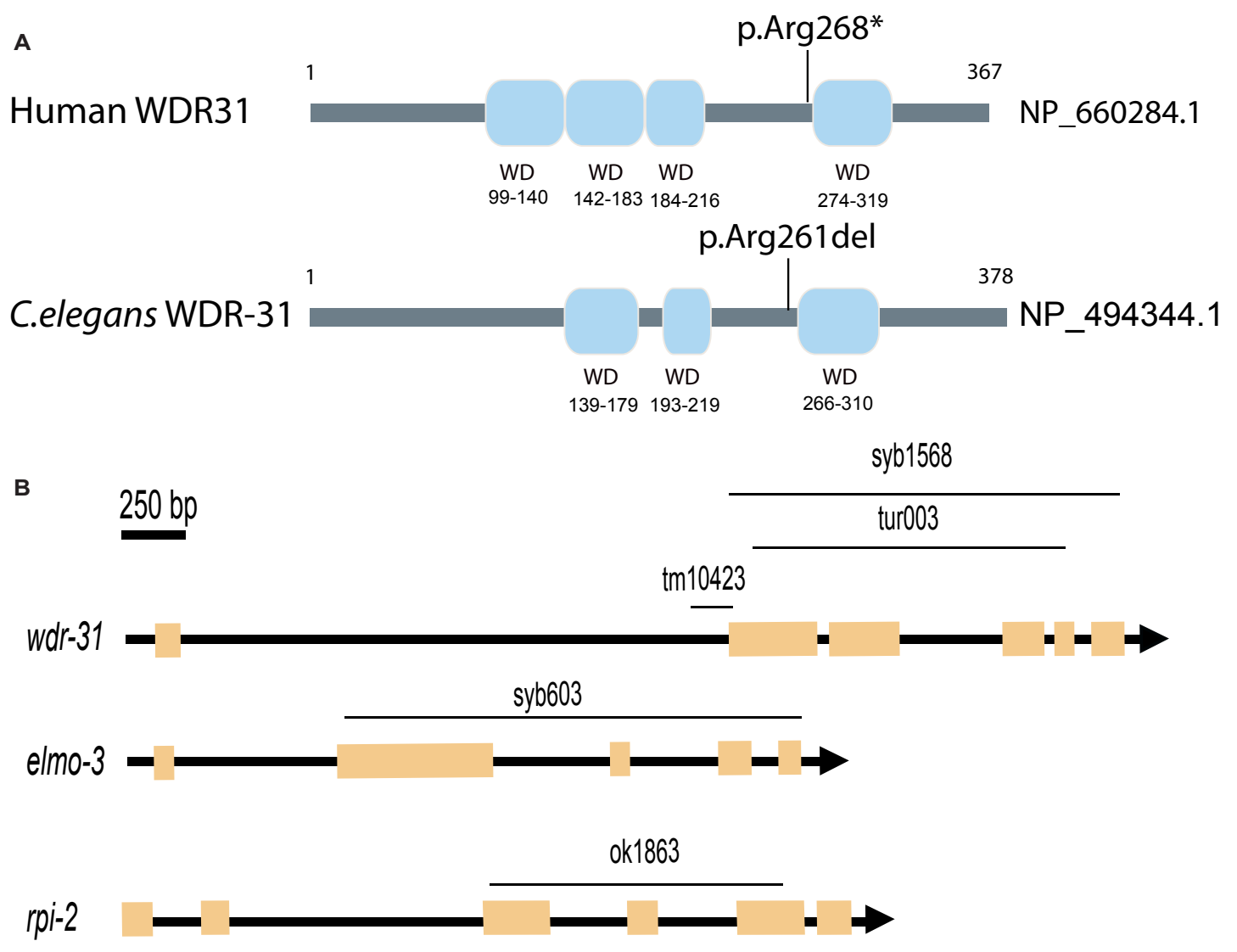

C

wdr-31(tm10423);rpi-2;elmd-1
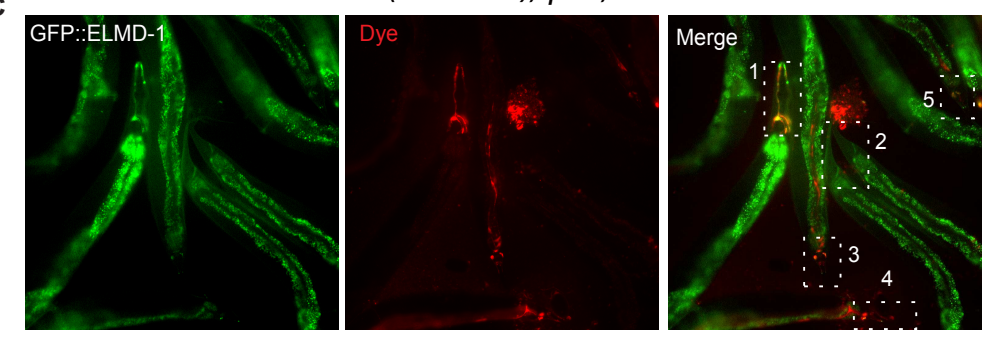

D RPI-2::GFP
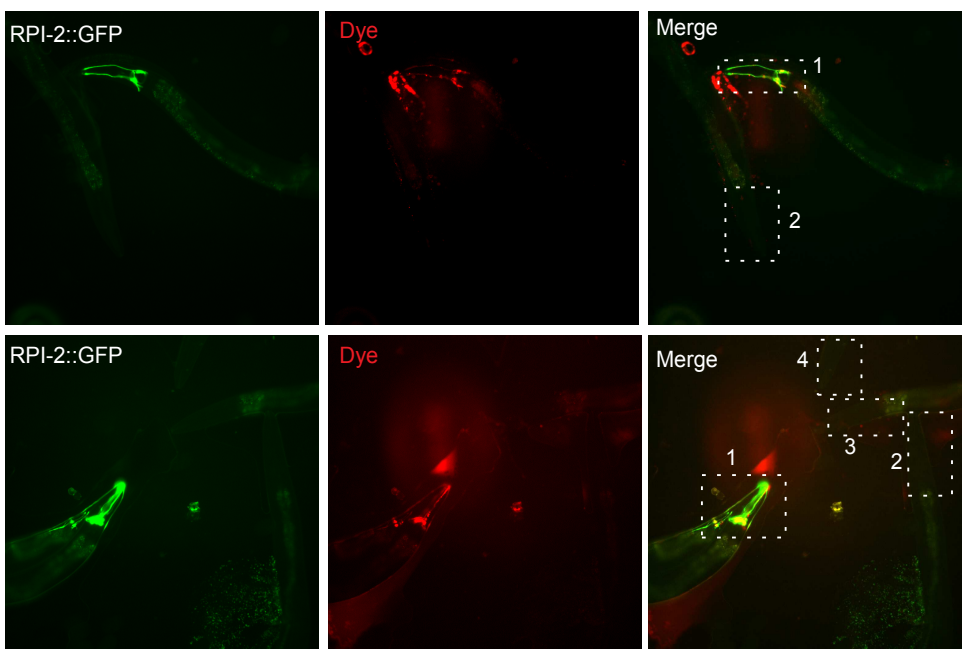


\section{Supplementary Figure 3: Rescue of dye uptake defects by ELMD-1 or RPI-2.}

836 A) Shown are representative schematics of human and C. elegans WDR-31/WDR31. Human 837 WDR31 (NP_660284.1) has four WD domains while C. elegans WDR-31 (NP_494344.1) has 838 three WD domains (https://prosite.expasy.org/; Sigrist CJA et al 2012). B) Schematic diagrams of $839 w d r-31, e l m d-1$ and $r p i-2$ together with corresponding deletions are shown. Scale bars: $250 \mathrm{bp} . \mathbf{C}$ 840 and D) Fluorescence microscope images of mutant worms (head and tail) with red-fluorescent dye 841 uptake (texas red filter) and GFP-tagged ELMD-1 and RPI-2 (fluorescence filter set for GFP) are

842 shown. The head or tail in the dotted lines is indicated by numbers in the combined fluorescence 843 images. GFP expression in combination with RED fluorescence indicates the rescue of dye uptake 844 defects.

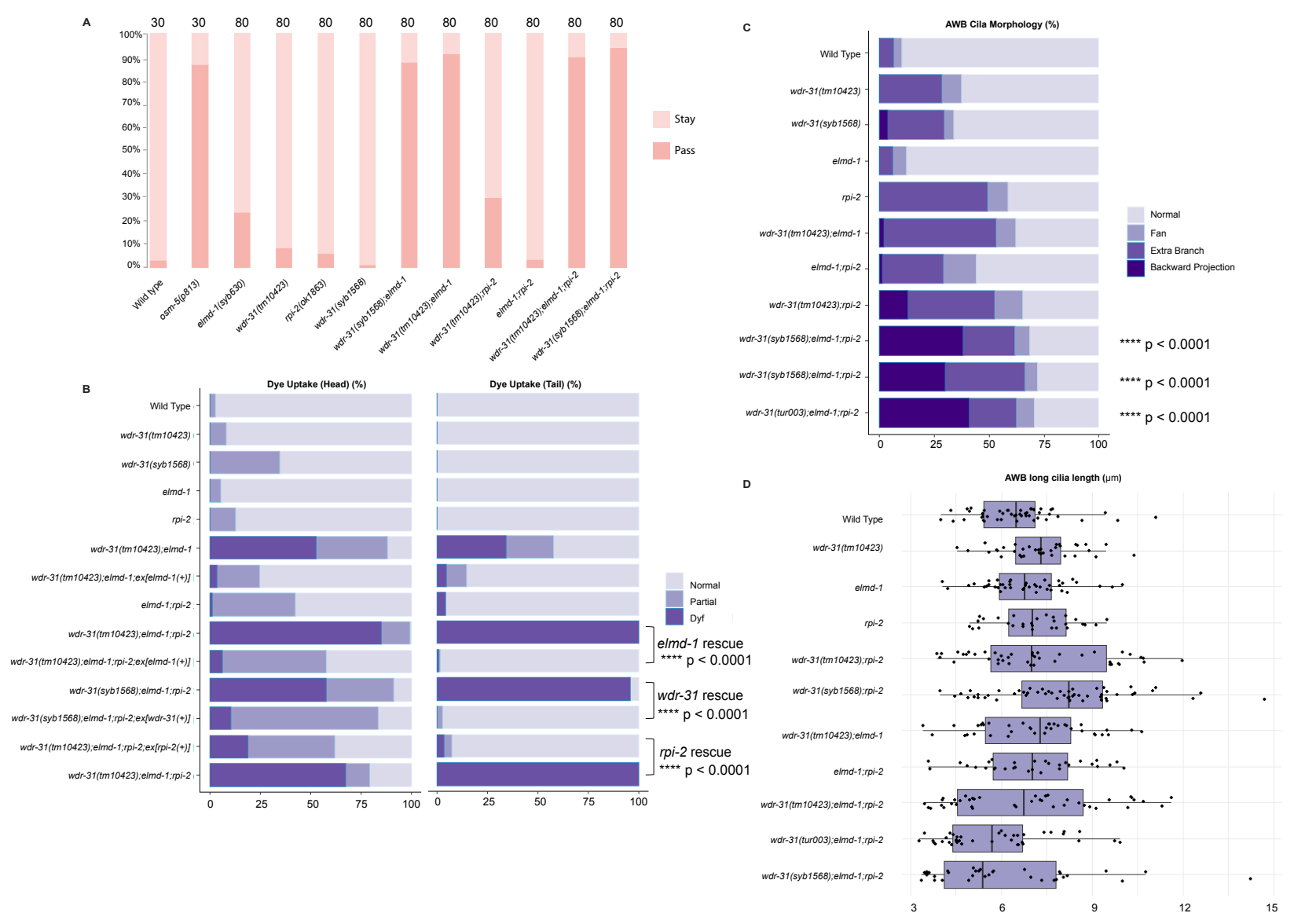


846 Supplementary Figure 4: A) 100\% stacked column chart displays the proportion of C. elegans

847 wild type and indicated mutants cross the osmotic barrier containing an $8 \mathrm{M}$ fructose solution with

848 bromophenol blue. Stay represents not crossing the osmotic barrier while pass means that animal

849 crossed the osmotic barrier. The number of animals used in osmotic avoidance assays for each

850 strain are shown on the bar charts. B and C) The percentage of dye uptake in the head and tail in

851 wild type and mutants, as well as the defect in AWB cilia morphology, are displayed in bar charts.

852 Dyf represents dye uptake defects while the partial means some cells in the head or tail take up the

853 fluorescence dye but others could not fill up their cells. Fisher's exact test was performed for

854 statistical analysis between indicated triple mutants and a rescue gene for Dye assay or wild type

855 and indicated mutants for AWB cilia morphology. Brackets show statistical significance between

856 two strains compared $(\mathrm{p}<0.0001)$ in B and **** indicates statistical significance in C. D) Shown

857 is the jitter plot for AWB long cilia length $(\mu \mathrm{m})$ for wild type and indicated mutant strains. 

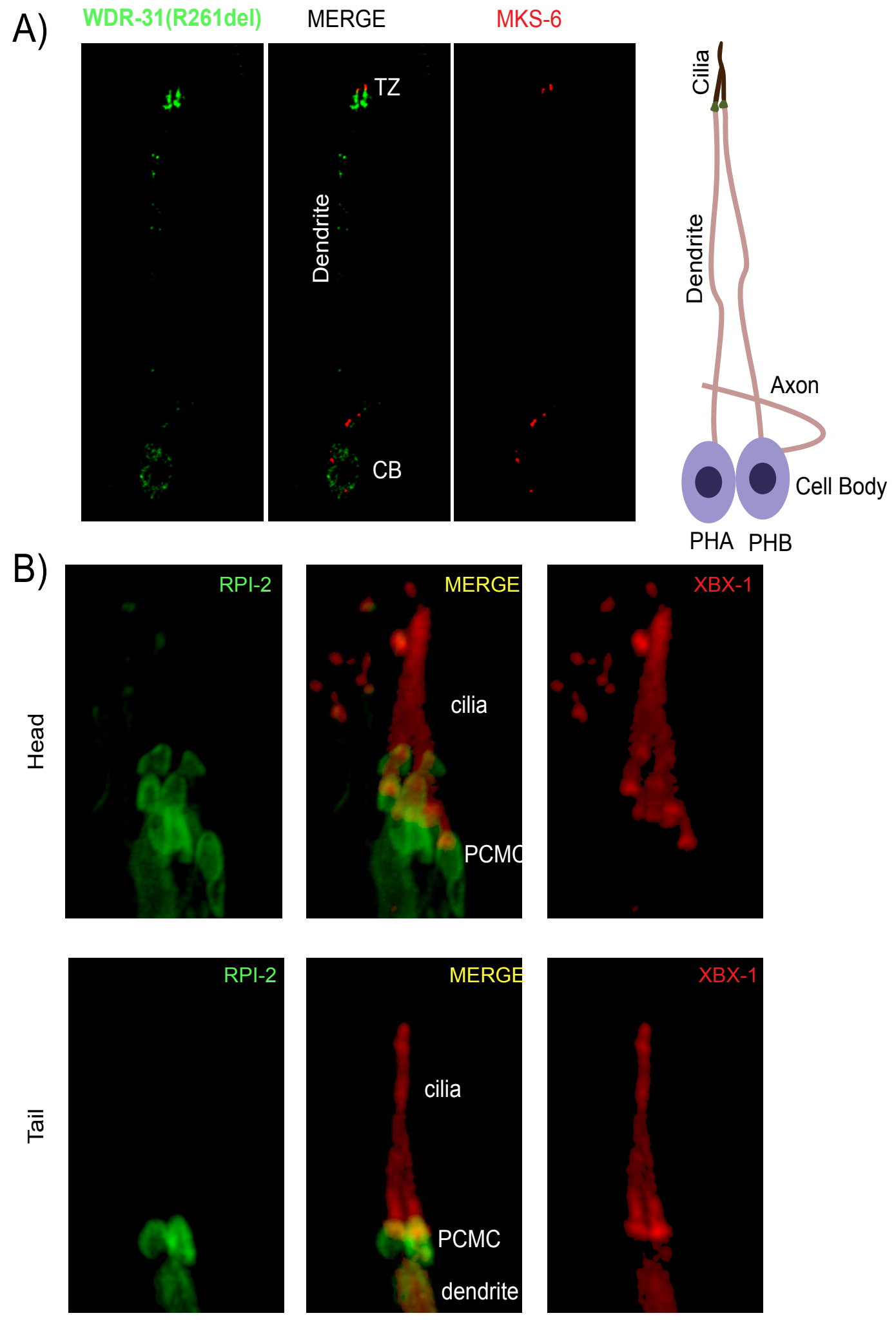
859 Supplementary Figure 5: A) Shown is the co-localization of WDR-31(R261del)::GFP (a $C$.

860 elegans variant mimicking disease-associated human R268* variant) and MKS-6::mCherry 861 (transition zone protein) in the tail (PHA/PHB sensory neuron) of C. elegans. Shown is a schematic 862 drawing of the PHA/PHB sensory neuron. CB denotes the cell body. axon dendrite and transition 863 zone are shown in the images and schematic drawing. B) Shown is co-localization of RPI-2::GFP 864 (human RP2) and XBX-1::mCherry (human DYNC2LI1) in the head and tail sensory neurons. 865 XBX-1 stains the entire cilium and is used as a ciliary marker while RPI-2 is located at PCMC. 866 PCMC denotes the periciliary membrane compartment. 

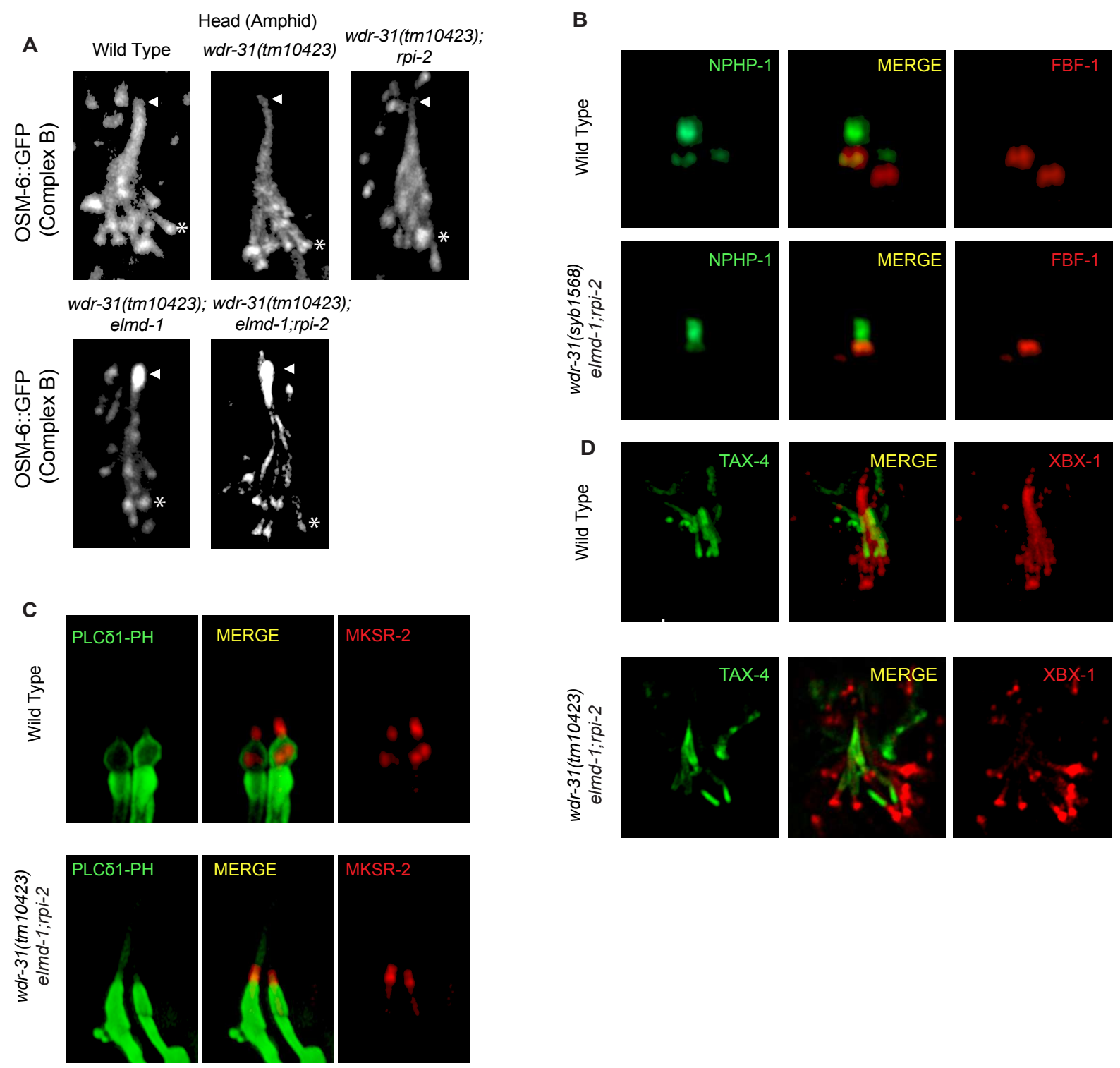

868 Supplementary Figure 6: A) Confocal images (Z-stack) display the localization of OSM-6::GFP

869 (a single copy transgene, huma IFT52) in the head (a bunch of cilia) of wild type and indicated

870 mutants. Arrow points the ciliary tips while asterisks indicate the ciliary base. B, C and D)

871 Confocal images show colocalization of TAX-4 (a ciliary membrane protein) and XBX-

$8721::$ tdTomato (a cilia marker) or NPHP-1::GFP (transition zone protein) and FBF-1:: mCherry

873 (transition fiber protein) or PLC 1 1-PH::GFP (a marker for monitoring phosphatidylinositol 4,5-

874 bisphosphate (PtdIns(4,5)P2) in the plasma membrane) and MKSR-2 (a TZ marker). The 
875 localization of TAX-4::GFP in $w d r-31$; elmd-1;rpi-2 triple mutants was similar to wild type. The 876 PLC 1 1-PH::GFP decorates the membranes of PCMC and does not enter into cilia in wild type.

877 The PLC $81-\mathrm{PH}::$ GFP stays outside of cilia in the $w d r-31$; elmd-1;rpi-2 triple mutants.

878

879 Supplementary Movies

880 For the IFT assay, time-lapse movies (3 frames per second) were generated with Leica DM6, and 881 were processed with Image J to generate GIFs (10 fps).

882 Supplementary Movies:

883 Supplementary Movie 1: IFT-74::GFP in wild type and indicated mutant backgrounds.

884 Supplementary Movie 2: OSM-6::GFP in wild type and indicated mutant backgrounds.

885 Supplementary Movie 3: OSM-3::GFP in wild type and indicated mutant backgrounds

886 Supplementary Movie 4: GFP::CHE-3 in wild type and indicated mutant backgrounds.

887 Supplementary Movie 5: IFT-140::GFP in wild type and indicated mutant backgrounds.

888 Supplementary Movie 6: BBS-7::GFP in wild type and indicated mutant backgrounds.

889 References

890 Ansley, S.J., Badano, J.L., Blacque, O.E., Hill, J., Hoskins, B.E., Leitch, C.C., Chul Kim, J., Ross, A.J., Eichers, E.R., Teslovich, T.M., Mah, A.K., Johnsen, R.C., Cavender, J.C., Alan Lewis, R., Leroux, M.R., Beales, P.L., Katsanis, N., 2003. Basal body dysfunction is a likely cause of pleiotropic Bardet-Biedl syndrome. Nature 425, 628-633. https://doi.org/10.1038/nature02030

Anvarian, Z., Mykytyn, K., Mukhopadhyay, S., Pedersen, L.B., Christensen, S.T., 2019. Cellular signalling by primary cilia in development, organ function and disease. Nat. Rev. Nephrol. 15, 199-219. https://doi.org/10.1038/s41581-019-0116-9

Arnaiz, O., Malinowska, A., Klotz, C., Sperling, L., Dadlez, M., Koll, F., Cohen, J., 2009. Cildb: a knowledgebase for centrosomes and cilia. Database 2009. https://doi.org/10.1093/database/bap022

Avidor-Reiss, T., Maer, A.M., Koundakjian, E., Polyanovsky, A., Keil, T., Subramaniam, S., Zuker, C.S., 2004. Decoding Cilia Function. Cell 117, 527-539. https://doi.org/10.1016/S0092-8674(04)00412-X 
908

Blacque, O., E., 2008. Intraflagellar transport: from molecular characterisation to mechanism. Front. Biosci. 13, 2633. https://doi.org/10.2741/2871

Blacque, O.E., 2004. Loss of C. elegans BBS-7 and BBS-8 protein function results in cilia defects and compromised intraflagellar transport. Genes Dev. 18, 1630-1642. https://doi.org/10.1101/gad.1194004

Blacque, O.E., Li, C., Inglis, P.N., Esmail, M.A., Ou, G., Mah, A.K., Baillie, D.L., Scholey, J.M., Leroux, M.R., 2006. The WD Repeat-containing Protein IFTA-1 Is Required for Retrograde Intraflagellar Transport. Mol. Biol. Cell 17, 5053-5062. https://doi.org/10.1091/mbc.e06-06-0571

Blacque, O.E., Perens, E.A., Boroevich, K.A., Inglis, P.N., Li, C., Warner, A., Khattra, J., Holt, R.A., Ou, G., Mah, A.K., McKay, S.J., Huang, P., Swoboda, P., Jones, S.J.M., Marra, M.A., Baillie, D.L., Moerman, D.G., Shaham, S., Leroux, M.R., 2005. Functional Genomics of the Cilium, a Sensory Organelle. Curr. Biol. 15, 935-941. https://doi.org/10.1016/j.cub.2005.04.059

Blacque, O.E., Sanders, A.A., 2014. Compartments within a compartment: What C. elegans can tell us about ciliary subdomain composition, biogenesis, function, and disease. Organogenesis 10, 126-137. https://doi.org/10.4161/org.28830

Bloodgood, R.A., 2009. From Central to Rudimentary to Primary: The History of an Underappreciated Organelle Whose Time Has Come.The Primary Cilium, in: Methods in Cell Biology. Elsevier, pp. 2-52. https://doi.org/10.1016/S0091-679X(08)94001-2

Brenner, S., 1974. The genetics of Caenorhabditis elegans. Genetics 77, 71-94.

Breslow, D.K., Hoogendoorn, S., Kopp, A.R., Morgens, D.W., Vu, B.K., Kennedy, M.C., Han, K., Li, A., Hess, G.T., Bassik, M.C., Chen, J.K., Nachury, M.V., 2018. A CRISPR-based screen for Hedgehog signaling provides insights into ciliary function and ciliopathies. Nat. Genet. 50, 460-471. https://doi.org/10.1038/s41588-018-0054-7

Chang, N., Sun, C., Gao, L., Zhu, D., Xu, X., Zhu, X., Xiong, J.-W., Xi, J.J., 2013. Genome editing with RNA-guided Cas9 nuclease in Zebrafish embryos. Cell Res. 23, 465-472. https://doi.org/10.1038/cr.2013.45

Choksi, S.P., Babu, D., Lau, D., Yu, X., Roy, S., 2014. Systematic discovery of novel ciliary genes through functional genomics in the zebrafish. Development 141, 3410-3419. https://doi.org/10.1242/dev.108209

Dickinson, D.J., Ward, J.D., Reiner, D.J., Goldstein, B., 2013. Engineering the Caenorhabditis elegans genome using Cas9-triggered homologous recombination. Nat. Methods 10, 1028-1034. https://doi.org/10.1038/nmeth.2641

Evans, R.J., Schwarz, N., Nagel-Wolfrum, K., Wolfrum, U., Hardcastle, A.J., Cheetham, M.E., 2010. The retinitis pigmentosa protein RP2 links pericentriolar vesicle transport between the Golgi and the primary cilium. Hum. Mol. Genet. 19, 1358-1367. https://doi.org/10.1093/hmg/ddq012

Gabler, F., Nam, S., Till, S., Mirdita, M., Steinegger, M., Söding, J., Lupas, A.N., Alva, V., 2020. 
Protein Sequence Analysis Using the MPI Bioinformatics Toolkit. Curr. Protoc. Bioinforma. 72. https://doi.org/10.1002/cpbi.108

Haycraft, C.J., Swoboda, P., Taulman, P.D., Thomas, J.H., Yoder, B.K., 2001. The C. elegans homolog of the murine cystic kidney disease gene Tg737 functions in a ciliogenic pathway and is disrupted in osm-5 mutant worms. Dev. Camb. Engl. 128, 1493-1505.

Herman, R.K., Hedgecock, E.M., 1990. Limitation of the size of the vulval primordium of Caenorhabditis elegans by lin-15 expression in surrounding hypodermis. Nature 348 , 169-171. https://doi.org/10.1038/348169a0

Ivanova, A.A., East, M.P., Yi, S.L., Kahn, R.A., 2014. Characterization of Recombinant ELMOD (Cell Engulfment and Motility Domain) Proteins as GTPase-activating Proteins (GAPs) for ARF Family GTPases. J. Biol. Chem. 289, 11111-11121. https://doi.org/10.1074/jbc.M114.548529

Jaworek, T.J., Richard, E.M., Ivanova, A.A., Giese, A.P.J., Choo, D.I., Khan, S.N., Riazuddin, Sheikh, Kahn, R.A., Riazuddin, Saima, 2013. An Alteration in ELMOD3, an Arl2 GTPase-Activating Protein, Is Associated with Hearing Impairment in Humans. PLoS Genet. 9, e1003774. https://doi.org/10.1371/journal.pgen.1003774

Jensen, V.L., Carter, S., Sanders, A.A.W.M., Li, C., Kennedy, J., Timbers, T.A., Cai, J., Scheidel, N., Kennedy, B.N., Morin, R.D., Leroux, M.R., Blacque, O.E., 2016. WholeOrganism Developmental Expression Profiling Identifies RAB-28 as a Novel Ciliary GTPase Associated with the BBSome and Intraflagellar Transport. PLOS Genet. 12, e1006469. https://doi.org/10.1371/journal.pgen.1006469

Jin, H., White, S.R., Shida, T., Schulz, S., Aguiar, M., Gygi, S.P., Bazan, J.F., Nachury, M.V., 2010. The Conserved Bardet-Biedl Syndrome Proteins Assemble a Coat that Traffics Membrane Proteins to Cilia. Cell 141, 1208-1219. https://doi.org/10.1016/j.cell.2010.05.015

Johnson, K.R., Longo-Guess, C.M., Gagnon, L.H., 2012. Mutations of the Mouse ELMO Domain Containing 1 Gene (Elmod1) Link Small GTPase Signaling to Actin Cytoskeleton Dynamics in Hair Cell Stereocilia. PLoS ONE 7, e36074. https://doi.org/10.1371/journal.pone.0036074

Kaplan, O.I., Doroquez, D.B., Cevik, S., Bowie, R.V., Clarke, L., Sanders, A.A.W.M., Kida, K., Rappoport, J.Z., Sengupta, P., Blacque, O.E., 2012. Endocytosis Genes Facilitate Protein and Membrane Transport in C. elegans Sensory Cilia. Curr. Biol. 22, 451-460. https://doi.org/10.1016/j.cub.2012.01.060

Lambacher, N.J., Bruel, A.-L., van Dam, T.J.P., Szymańska, K., Slaats, G.G., Kuhns, S., McManus, G.J., Kennedy, J.E., Gaff, K., Wu, K.M., van der Lee, R., Burglen, L., Doummar, D., Rivière, J.-B., Faivre, L., Attié-Bitach, T., Saunier, S., Curd, A., Peckham, M., Giles, R.H., Johnson, C.A., Huynen, M.A., Thauvin-Robinet, C., Blacque, O.E., 2016. TMEM107 recruits ciliopathy proteins to subdomains of the ciliary transition zone and causes Joubert syndrome. Nat. Cell Biol. 18, 122-131. https://doi.org/10.1038/ncb3273

Lechtreck, K.-F., Johnson, E.C., Sakai, T., Cochran, D., Ballif, B.A., Rush, J., Pazour, G.J., Ikebe, M., Witman, G.B., 2009. The Chlamydomonas reinhardtii BBSome is an IFT 
1041

1042

1043

1044

1045

1046

1047

1048

1049

1050

1051

1052

1053

1054

1055

1056

1057

1058

1059

1060

cargo required for export of specific signaling proteins from flagella. J. Cell Biol. 187, 1117-1132. https://doi.org/10.1083/jcb.200909183

Lee, E., Sivan-Loukianova, E., Eberl, D.F., Kernan, M.J., 2008. An IFT-A protein is required to delimit functionally distinct zones in mechanosensory cilia. Curr. Biol. CB 18, 18991906. https://doi.org/10.1016/j.cub.2008.11.020

Li, J.B., Gerdes, J.M., Haycraft, C.J., Fan, Y., Teslovich, T.M., May-Simera, H., Li, H., Blacque, O.E., Li, L., Leitch, C.C., Lewis, R.A., Green, J.S., Parfrey, P.S., Leroux, M.R., Davidson, W.S., Beales, P.L., Guay-Woodford, L.M., Yoder, B.K., Stormo, G.D., Katsanis, N., Dutcher, S.K., 2004. Comparative Genomics Identifies a Flagellar and Basal Body Proteome that Includes the BBS5 Human Disease Gene. Cell 117, 541-552. https://doi.org/10.1016/S0092-8674(04)00450-7

Liem, K.F., Ashe, A., He, M., Satir, P., Moran, J., Beier, D., Wicking, C., Anderson, K.V., 2012. The IFT-A complex regulates Shh signaling through cilia structure and membrane protein trafficking. J. Cell Biol. 197, 789-800. https://doi.org/10.1083/jcb.201110049

Loktev, A.V., Zhang, Q., Beck, J.S., Searby, C.C., Scheetz, T.E., Bazan, J.F., Slusarski, D.C., Sheffield, V.C., Jackson, P.K., Nachury, M.V., 2008. A BBSome Subunit Links Ciliogenesis, Microtubule Stability, and Acetylation. Dev. Cell 15, 854-865. https://doi.org/10.1016/j.devcel.2008.11.001

Mangeol, P., Prevo, B., Peterman, E.J.G., 2016. KymographClear and KymographDirect: two tools for the automated quantitative analysis of molecular and cellular dynamics using kymographs. Mol. Biol. Cell 27, 1948-1957. https://doi.org/10.1091/mbc.E15-06-0404

Mick, D.U., Rodrigues, R.B., Leib, R.D., Adams, C.M., Chien, A.S., Gygi, S.P., Nachury, M.V., 2015. Proteomics of Primary Cilia by Proximity Labeling. Dev. Cell 35, 497-512. https://doi.org/10.1016/j.devcel.2015.10.015

Miryounesi, M., Bahari, S., Salehpour, S., Alipour, N., Ghafouri-Fard, S., 2019. ELMO Domain Containing 1 (ELMOD1) Gene Mutation Is Associated with Mental Retardation and Autism Spectrum Disorder. J. Mol. Neurosci. 69, 312-315. https://doi.org/10.1007/s12031-019-01359-z

Mukhopadhyay, S., Wen, X., Chih, B., Nelson, C.D., Lane, W.S., Scales, S.J., Jackson, P.K., 2010. TULP3 bridges the IFT-A complex and membrane phosphoinositides to promote trafficking of $\mathrm{G}$ protein-coupled receptors into primary cilia. Genes Dev. 24, 2180-2193. https://doi.org/10.1101/gad.1966210

Nachury, M.V., 2014. How do cilia organize signalling cascades? Philos. Trans. R. Soc. Lond. B. Biol. Sci. 369. https://doi.org/10.1098/rstb.2013.0465

Nachury, M.V., Loktev, A.V., Zhang, Q., Westlake, C.J., Peränen, J., Merdes, A., Slusarski, D.C., Scheller, R.H., Bazan, J.F., Sheffield, V.C., Jackson, P.K., 2007. A Core Complex of BBS Proteins Cooperates with the GTPase Rab8 to Promote Ciliary Membrane Biogenesis. Cell 129, 1201-1213. https://doi.org/10.1016/j.cell.2007.03.053

Nozaki, S., Castro Araya, R.F., Katoh, Y., Nakayama, K., 2019. Requirement of IFT-B-BBSome complex interaction in export of GPR161 from cilia. Biol. Open 8, bio043786. 
Ou, G., E. Blacque, O., Snow, J.J., Leroux, M.R., Scholey, J.M., 2005. Functional coordination of intraflagellar transport motors. Nature 436, 583-587. https://doi.org/10.1038/nature03818

Ou, G., Koga, M., Blacque, O.E., Murayama, T., Ohshima, Y., Schafer, J.C., Li, C., Yoder, B.K., Leroux, M.R., Scholey, J.M., 2007. Sensory Ciliogenesis in Caenorhabditis elegans: Assignment of IFT Components into Distinct Modules Based on Transport and Phenotypic Profiles. Mol. Biol. Cell 18, 1554-1569. https://doi.org/10.1091/mbc.e06-090805

Patel-King, R.S., Gilberti, R.M., Hom, E.F.Y., King, S.M., 2013. WD60/FAP163 is a dynein intermediate chain required for retrograde intraflagellar transport in cilia. Mol. Biol. Cell 24, 2668-2677. https://doi.org/10.1091/mbc.E13-05-0266

Pazour, G.J., Dickert, B.L., Vucica, Y., Seeley, E.S., Rosenbaum, J.L., Witman, G.B., Cole, D.G., 2000. Chlamydomonas IFT88 and its mouse homologue, polycystic kidney disease gene $\operatorname{tg} 737$, are required for assembly of cilia and flagella. J. Cell Biol. 151, 709-718. https://doi.org/10.1083/jcb.151.3.709

Piasecki, B.P., Burghoorn, J., Swoboda, P., 2010. Regulatory Factor X (RFX)-mediated transcriptional rewiring of ciliary genes in animals. Proc. Natl. Acad. Sci. 107, 1296912974. https://doi.org/10.1073/pnas.0914241107

Prevo, B., Scholey, J.M., Peterman, E.J.G., 2017. Intraflagellar transport: mechanisms of motor action, cooperation, and cargo delivery. FEBS J. 284, 2905-2931. https://doi.org/10.1111/febs.14068

Reiter, J.F., Leroux, M.R., 2017. Genes and molecular pathways underpinning ciliopathies. Nat. Rev. Mol. Cell Biol. 18, 533-547. https://doi.org/10.1038/nrm.2017.60

Richards, S., Aziz, N., Bale, S., Bick, D., Das, S., Gastier-Foster, J., Grody, W.W., Hegde, M., Lyon, E., Spector, E., Voelkerding, K., Rehm, H.L., ACMG Laboratory Quality Assurance Committee, 2015. Standards and guidelines for the interpretation of sequence variants: a joint consensus recommendation of the American College of Medical Genetics and Genomics and the Association for Molecular Pathology. Genet. Med. Off. J. Am. Coll. Med. Genet. 17, 405-424. https://doi.org/10.1038/gim.2015.30

Rompolas, P., Pedersen, L.B., Patel-King, R.S., King, S.M., 2007. Chlamydomonas FAP133 is a dynein intermediate chain associated with the retrograde intraflagellar transport motor. J. Cell Sci. 120, 3653-3665. https://doi.org/10.1242/jcs.012773

Rosenbaum, J.L., Witman, G.B., 2002. Intraflagellar transport. Nat. Rev. Mol. Cell Biol. 3, 813825. https://doi.org/10.1038/nrm952

Ruiz García, S., Deprez, M., Lebrigand, K., Cavard, A., Paquet, A., Arguel, M.-J., Magnone, V., Truchi, M., Caballero, I., Leroy, S., Marquette, C.-H., Marcet, B., Barbry, P., Zaragosi, L.E., 2019. Novel dynamics of human mucociliary differentiation revealed by single-cell RNA sequencing of nasal epithelial cultures. Development dev.177428. 
Satir, P., Christensen, S.T., 2007. Overview of Structure and Function of Mammalian Cilia. Annu. Rev. Physiol. 69, 377-400. https://doi.org/10.1146/annurev.physiol.69.040705.141236

Saudi Mendeliome Group, 2015. Comprehensive gene panels provide advantages over clinical exome sequencing for Mendelian diseases. Genome Biol. 16, 134. https://doi.org/10.1186/s13059-015-0693-2

Schneider, C.A., Rasband, W.S., Eliceiri, K.W., 2012. NIH Image to ImageJ: 25 years of image analysis. Nat. Methods 9, 671-675. https://doi.org/10.1038/nmeth.2089

Scholey, J., 2007. The sensory cilia of Caenorhabditis elegans_Revised. WormBook. https://doi.org/10.1895/wormbook.1.126.2

Schwarz, N., Lane, A., Jovanovic, K., Parfitt, D.A., Aguila, M., Thompson, C.L., da Cruz, L., Coffey, P.J., Chapple, J.P., Hardcastle, A.J., Cheetham, M.E., 2017. Arl3 and RP2 regulate the trafficking of ciliary tip kinesins. Hum. Mol. Genet. 26, 2480-2492. https://doi.org/10.1093/hmg/ddx143

Shaheen, R., Szymanska, K., Basu, B., Patel, N., Ewida, N., Faqeih, E., Al Hashem, A., Derar, N., Alsharif, H., Aldahmesh, M.A., Alazami, A.M., Hashem, M., Ibrahim, N., Abdulwahab, F.M., Sonbul, R., Alkuraya, H., Alnemer, M., Al Tala, S., Al-Husain, M., Morsy, H., Seidahmed, M.Z., Meriki, N., Al-Owain, M., AlShahwan, S., Tabarki, B., Salih, M.A., Ciliopathy WorkingGroup, Faquih, T., El-Kalioby, M., Ueffing, M., Boldt, K., Logan, C.V., Parry, D.A., Al Tassan, N., Monies, D., Megarbane, A., Abouelhoda, M., Halees, A., Johnson, C.A., Alkuraya, F.S., 2016. Characterizing the morbid genome of ciliopathies. Genome Biol. 17, 242. https://doi.org/10.1186/s13059-016-1099-5

Shamseldin, H.E., Shaheen, R., Ewida, N., Bubshait, D.K., Alkuraya, H., Almardawi, E., Howaidi, A., Sabr, Y., Abdalla, E.M., Alfaifi, A.Y., Alghamdi, J.M., Alsagheir, A., Alfares, A., Morsy, H., Hussein, M.H., Al-Muhaizea, M.A., Shagrani, M., Al Sabban, E., Salih, M.A., Meriki, N., Khan, R., Almugbel, M., Qari, A., Tulba, M., Mahnashi, M., Alhazmi, K., Alsalamah, A.K., Nowilaty, S.R., Alhashem, A., Hashem, M., Abdulwahab, F., Ibrahim, N., Alshidi, T., AlObeid, E., Alenazi, M.M., Alzaidan, H., Rahbeeni, Z., Al-Owain, M., Sogaty, S., Seidahmed, M.Z., Alkuraya, F.S., 2020. The morbid genome of ciliopathies: an update. Genet. Med. Off. J. Am. Coll. Med. Genet. 22, 1051-1060. https://doi.org/10.1038/s41436-020-0761-1

Sigg, M.A., Menchen, T., Lee, C., Johnson, J., Jungnickel, M.K., Choksi, S.P., Garcia, G., Busengdal, H., Dougherty, G.W., Pennekamp, P., Werner, C., Rentzsch, F., Florman, H.M., Krogan, N., Wallingford, J.B., Omran, H., Reiter, J.F., 2017. Evolutionary Proteomics Uncovers Ancient Associations of Cilia with Signaling Pathways. Dev. Cell 43, 744-762.e11. https://doi.org/10.1016/j.devcel.2017.11.014

Silflow, C.D., Lefebvre, P.A., 2001. Assembly and Motility of Eukaryotic Cilia and Flagella. Lessons from Chlamydomonas reinhardtii. Plant Physiol. 127, 1500-1507. https://doi.org/10.1104/pp.010807 
Sleigh, M.A., 1989. Adaptations of ciliary systems for the propulsion of water and mucus. Comp. Biochem. Physiol. A Physiol. 94, 359-364. https://doi.org/10.1016/0300-9629(89)905598

SYSCILIA Study Group, van Dam, T.J., Wheway, G., Slaats, G.G., Huynen, M.A., Giles, R.H., 2013. The SYSCILIA gold standard (SCGSv1) of known ciliary components and its applications within a systems biology consortium. Cilia 2, 7 . https://doi.org/10.1186/2046-2530-2-7

Thisse, B., Heyer, V., Lux, A., Alunni, V., Degrave, A., Seiliez, I., Kirchner, J., Parkhill, J.-P., Thisse, C., 2004. Spatial and Temporal Expression of the Zebrafish Genome by LargeScale In Situ Hybridization Screening, in: Methods in Cell Biology. Elsevier, pp. 505519. https://doi.org/10.1016/S0091-679X(04)77027-2

Turn, R.E., Linnert, J., Gigante, E.D., Wolfrum, U., Caspary, T., Kahn, R.A., 2021. Roles for ELMOD2 and Rootletin in ciliogenesis. Mol. Biol. Cell 32, 800-822. https://doi.org/10.1091/mbc.E20-10-0635

UK10K Rare Diseases Group, Boldt, K., van Reeuwijk, J., Lu, Q., Koutroumpas, K., Nguyen, T.M.T., Texier, Y., van Beersum, S.E.C., Horn, N., Willer, J.R., Mans, D.A., Dougherty, G., Lamers, I.J.C., Coene, K.L.M., Arts, H.H., Betts, M.J., Beyer, T., Bolat, E., Gloeckner, C.J., Haidari, K., Hetterschijt, L., laconis, D., Jenkins, D., Klose, F., Knapp, B., Latour, B., Letteboer, S.J.F., Marcelis, C.L., Mitic, D., Morleo, M., Oud, M.M., Riemersma, M., Rix, S., Terhal, P.A., Toedt, G., van Dam, T.J.P., de Vrieze, E., Wissinger, Y., Wu, K.M., Apic, G., Beales, P.L., Blacque, O.E., Gibson, T.J., Huynen, M.A., Katsanis, N., Kremer, H., Omran, H., van Wijk, E., Wolfrum, U., Kepes, F., Davis, E.E., Franco, B., Giles, R.H., Ueffing, M., Russell, R.B., Roepman, R., 2016. An organelle-specific protein landscape identifies novel diseases and molecular mechanisms. Nat. Commun. 7, 11491. https://doi.org/10.1038/ncomms11491

van Dam, T.J.P., Kennedy, J., van der Lee, R., de Vrieze, E., Wunderlich, K.A., Rix, S., Dougherty, G.W., Lambacher, N.J., Li, C., Jensen, V.L., Leroux, M.R., Hjeij, R., Horn, N., Texier, Y., Wissinger, Y., van Reeuwijk, J., Wheway, G., Knapp, B., Scheel, J.F., Franco, B., Mans, D.A., van Wijk, E., Képès, F., Slaats, G.G., Toedt, G., Kremer, H., Omran, H., Szymanska, K., Koutroumpas, K., Ueffing, M., Nguyen, T.-M.T., Letteboer, S.J.F., Oud, M.M., van Beersum, S.E.C., Schmidts, M., Beales, P.L., Lu, Q., Giles, R.H., Szklarczyk, R., Russell, R.B., Gibson, T.J., Johnson, C.A., Blacque, O.E., Wolfrum, U., Boldt, K., Roepman, R., Hernandez-Hernandez, V., Huynen, M.A., 2019. CiliaCarta: An integrated and validated compendium of ciliary genes. PLOS ONE 14, e0216705. https://doi.org/10.1371/journal.pone.0216705

Wei, Q., Xu, Q., Zhang, Y., Li, Y., Zhang, Q., Hu, Z., Harris, P.C., Torres, V.E., Ling, K., Hu, J., 2013. Transition fibre protein FBF1 is required for the ciliary entry of assembled intraflagellar transport complexes. Nat. Commun. 4, 2750. https://doi.org/10.1038/ncomms3750

Wei, Q., Zhang, Y., Li, Y., Zhang, Q., Ling, K., Hu, J., 2012. The BBSome controls IFT assembly and turnaround in cilia. Nat. Cell Biol. 14, 950-957. https://doi.org/10.1038/ncb2560

Wheway, G., Genomics England Research Consortium, Mitchison, H.M., 2019. Opportunities and Challenges for Molecular Understanding of Ciliopathies-The 100,000 Genomes 
Williams, C.L., Li, C., Kida, K., Inglis, P.N., Mohan, S., Semenec, L., Bialas, N.J., Stupay, R.M., Chen, N., Blacque, O.E., Yoder, B.K., Leroux, M.R., 2011. MKS and NPHP modules cooperate to establish basal body/transition zone membrane associations and ciliary gate function during ciliogenesis. J. Cell Biol. 192, 1023-1041. https://doi.org/10.1083/jcb.201012116

Williams, C.L., Mclntyre, J.C., Norris, S.R., Jenkins, P.M., Zhang, L., Pei, Q., Verhey, K., Martens, J.R., 2014. Direct evidence for BBSome-associated intraflagellar transport reveals distinct properties of native mammalian cilia. Nat. Commun. 5, 5813. https://doi.org/10.1038/ncomms6813

Wright, K.J., Baye, L.M., Olivier-Mason, A., Mukhopadhyay, S., Sang, L., Kwong, M., Wang, W., Pretorius, P.R., Sheffield, V.C., Sengupta, P., Slusarski, D.C., Jackson, P.K., 2011. An ARL3-UNC119-RP2 GTPase cycle targets myristoylated NPHP3 to the primary cilium. Genes Dev. 25, 2347-2360. https://doi.org/10.1101/gad.173443.111

Xu, Q., Zhang, Y., Wei, Q., Huang, Y., Li, Y., Ling, K., Hu, J., 2015. BBS4 and BBS5 show functional redundancy in the BBSome to regulate the degradative sorting of ciliary sensory receptors. Sci. Rep. 5, 11855. https://doi.org/10.1038/srep11855

Xu, W., Jin, M., Hu, R., Wang, H., Zhang, F., Yuan, S., Cao, Y., 2017. The Joubert Syndrome Protein Inpp5e Controls Ciliogenesis by Regulating Phosphoinositides at the Apical Membrane. J. Am. Soc. Nephrol. 28, 118-129. https://doi.org/10.1681/ASN.2015080906

Ye, F., Nager, A.R., Nachury, M.V., 2018. BBSome trains remove activated GPCRs from cilia by enabling passage through the transition zone. J. Cell Biol. 217, 1847-1868. https://doi.org/10.1083/jcb.201709041 


\section{Supplementary Files}

This is a list of supplementary files associated with this preprint. Click to download.

- SupplFigures.zip

- SuppleMovies.zip 